This postprint is published in:

Future Medicinal Chemistry, Vol. 10, No. 10

DOI 10.4155/fmc-2017-0257

\title{
Recent progress in the discovery and development of DNA gyrase B inhibitors
}

Michaela Barančoková, Danijel Kikelj, Janez Ilaš

Faculty of Pharmacy, University of Ljubljana, Aškerčeva 7, 1000 Ljubljana, Slovenia

\begin{abstract}
New antibacterials that modulate less explored targets are needed to fight the emerging bacterial resistance. DNA gyrase and topoisomerase IV are attractive targets in this search. These are both type II topoisomerases that can cleave both DNA strands, and can thus alter DNA topology during replication or similar processes. Currently, there are no ATP-competitive inhibitors of these two enzymes on the market, as the only aminocoumarin representative, novobiocin, was withdrawn due to safety concerns. The search for novel ATP-competitive inhibitors is a focus of ongoing industrial and academical research. This review summarises the recent efforts in the design, synthesis and evaluation of GyrB/ParE inhibitors. The various approaches to achieve improved antibacterial activities are described, with particular reference to Gram-negative bacteria.
\end{abstract}

Key words: antibacterial, topoisomerase II, DNA gyrase, topoisomerase IV, GyrB, ParE, ATPcompetitive, inhibitor, drug discovery, Gram-negative bacteria

\section{Graphical abstract:}






\section{Introduction}

The rapid progress in the field of antibacterial drug discovery that has occurred over previous decades significantly influenced the guidelines for treatment of bacterial infections. However, while the majority of antibacterial discoveries were made around the middle of the $20^{\text {th }}$ century, during what is often referred to as the "golden era", we are now facing the alarming threat of the "resistance era" [1]. Commonly accessible antibiotics have been taken for granted, and together with their misuse and/or overuse, this has now brought us to an era of increasing bacterial resistance [2]. This has been accompanied by a 'discovery void' of antibacterials over the last two decades [3, 4]. Nevertheless, many studies in industry and academia continue to investigate this field using modern approaches to overcome the problem of bacterial resistance, particularly through multi-targeting as a novel promising tactic that is under the spotlight in antibacterial drug discovery [5].

\section{DNA gyrase - structure, function and brief history of inhibitors from natural sources}

The topological state of DNA in the cell is altered by the topoisomerases, which are vital enzymes that support DNA replication and chromosome decatenation and condensation. The discovery of the first DNA topoisomerase by James Wang in 1971 led to rapid progress in this field, and was followed by the discovery of Escherichia coli DNA gyrase in 1976 [6, 7]. Since then, both DNA gyrase and the structurally related topoisomerase IV have been investigated in depth. Furthermore, as highly conserved enzymes for which the crystal structures are known, they are today recognised as well-established antibacterial targets.

DNA gyrase and topoisomerase IV are type II topoisomerases that have structural and functional similarities. DNA gyrase introduces negative supercoils into DNA in front of the replication fork, while topoisomerase IV is important for chromosome decatenation during DNA replication. Both of these enzymes comprise two subunits in a heterotetrameric structure, as $A_{2} B_{2}$ for DNA gyrase, and $C_{2} E_{2}$ for topoisomerase IV. The GyrA and ParC subunits introduce double-strand breaks into the DNA molecule, and thus release the torsional stress caused by supercoiling, with both strands then rejoined. GyrB and ParE contain an ATP-binding site, whereby ATP hydrolysis provides the energy required for their enzyme activities [8]. The similarities in the structures of DNA gyrase and topoisomerase IV offer an exceptional opportunity for dual targeting with new antibacterial compounds, thereby reducing the probability that the bacteria can develop target-based resistance against these actions.

DNA gyrase and topoisomerase IV belong to the wider group of Gyrase, Hsp90, Histidine Kinase, MutL (GHKL) proteins. These form an ATPase/kinase superfamily with an evolutionarily conserved protein domain and a similar ATP-binding fold across these diverse proteins, which as the name suggests, include DNA topoisomerase II, Hsp90 molecular chaperones, MutL DNA-mismatchrepair enzymes, and histidine kinases [9]. The structural similarity of the ATP binding site among these GHKL proteins can pose an obstacle for the design of selective ligands, although, on the other hand, 
ligands that target GyrB have been successfully used as starting points for the discovery of novel selective C-terminal Hsp90 inhibitors [10-12]. Novobiocin is a classical DNA GyrB inhibitor, and it was successfully modified to provide potent Hsp90 inhibitors with good anti-proliferative activities against multiple cancer cell lines. Other examples include marine alkaloid oroidin analogues, which are synthetic compounds with antiviral activities that target Hsp90 [13].

Gyrase poisons are inhibitors that target the GyrA and ParC subunits of DNA gyrase and topoisomerase IV, respectively. They stabilize the DNA-enzyme complex and prevent the DNA strands from rejoining. The introduction of DNA breaks can lead to cell death. The first representative described to have such a mechanism of action was nalidixic acid, which served as the template for optimization that led to the fluoroquinolone class of antibiotics. Indeed, many GyrA inhibitors are in clinical use (e.g., ciprofloxacin, gemifloxacin, levofloxacin, moxifloxacin, ofloxacin), although their resistance issues are rapidly worsening [14].

On the other hand, the situation for GyrB/ParC inhibitors is more complex. Novobiocin is a representative of the aminocoumarin class (Fig. 1), and it is the only GyrB inhibitor that has been put on the market. It was discovered through microbial screening of natural products in the 1950s, as a consequence of intensive research into antibacterial compounds. Its analogues clorobiocin and coumermycin $\mathrm{A}_{1}$ were discovered shortly afterwards (Fig. 1). At that time, penicillin was becoming less effective against the main pathogens, such as Staphylococcus aureus, and suitable replacements were desperately needed. Novobiocin was marketed as Albamycin ${ }^{\circledR}$, and its mainly Gram-positive spectrum of action was used against penicillin-resistant S. aureus infections. However, problems with limited solubility and eukaryotic toxicity soon occurred. Moreover, the availability of new types of safer drugs with greater efficacies (e.g., cephalosporines, penicillins with stability against penicillinase) resulted in reduced use of novobiocin during the 1960s, although it was not officially withdrawn from the market until 2011 [15].

Despite many efforts during the following years, no other ATP-competitive inhibitors of DNA gyrase have proven successful through phase I clinical studies [16]. However, as DNA gyrase is essential in bacteria but is not found in human cells, it remains a valuable drug target. Its ATP binding site is highly conserved, and the risk of the development of spontaneous resistance against ATP-competitive inhibitors is low. This thus provides the driving force for the present level of intense research into ATPcompetitive GyrB inhibitors. 


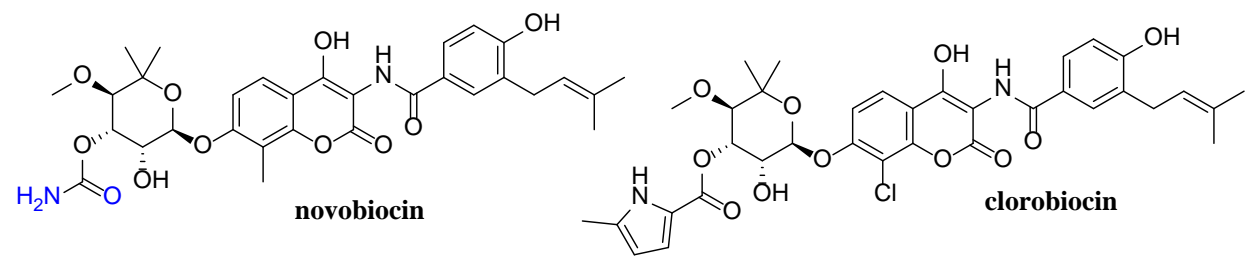

A



B<smiles>[R]N[C@H]([13CH])C([R])=O</smiles>

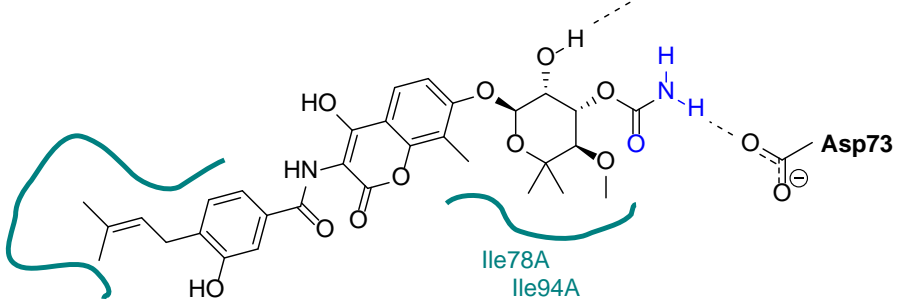

Pro79A

C<smiles>Cc1c(O)cc(O)c(CSC[C@H](NC(=O)[C@H](C)NC(=O)[C@H]2C(O)CCN2C(=O)[C@H](N)CO)C(=O)O)c1C(=O)O</smiles>

cyclothialidine

D

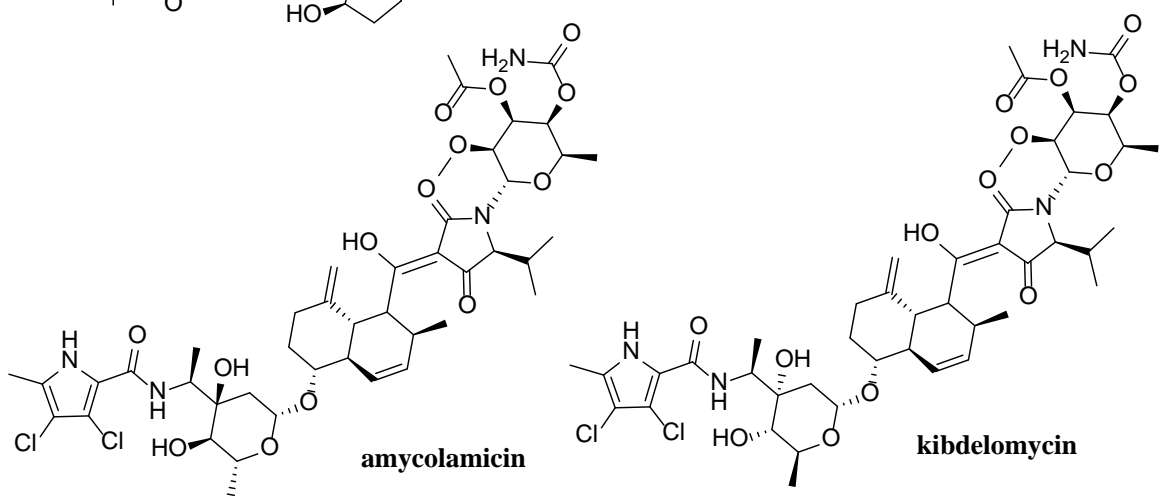

E

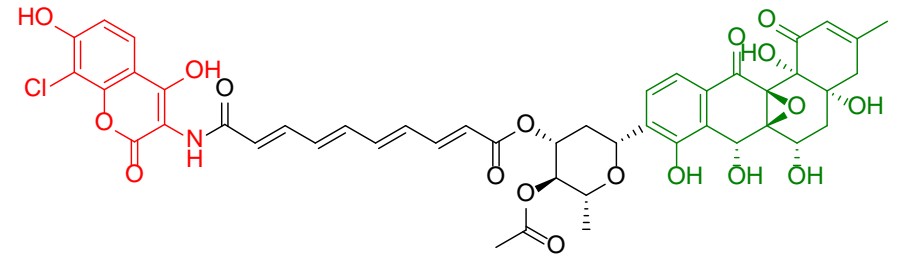

simocyclinone D8

Figure 1. (A) The aminocoumarin antibiotics novobiocin, clorobiocin and coumermycin A1. (B) The interactions between novobiocin and the amino-acid residues of the 24-kDa fragment of GyrB (PDB: 1AJ6) [17]. (C) Structure of cyclothialidine [18]. (D) Structure of amycolamicin and kibdelomycin. (E) Structure of simocyclinone D8, with its aminocoumarin moiety (red) and angucyclinone moiety (green) [19]. 
The structural and mechanistic features of DNA gyrase were discovered through the solving of the crystal structure of its $43-\mathrm{kDa} N$-terminal GyrB domain complexed with 5'-adenylyl- $\beta-\gamma-$ imidodiphosphate, which is a non-hydrolysable analogue of ATP (PDB: 1EI1) [20]. Later, the binding modes of the aminocoumarins were described based on the crystal structures of complexes between the 24-kDa N-terminal domain of E. coli GyrB and novobiocin (Fig. 1B), 5'-adenylyl- $\beta-\gamma-$ imidodiphosphate, and GR122222X (a representative of the cyclothialidine family). Comparative studies showed that the binding pockets of these structures partially overlap, which explains their ATPcompetitive inhibition even though they are structurally different [21].

Another group of natural products that act as gyrase inhibitors are the cyclothialidines (Fig. 1C) [18], which are also produced by Streptomyces spp. An ATP-competitive mode of inhibition by the cyclothialidines was shown, as for novobiocin [21]. The resorcinol ring of the cyclothialidines is involved in similar binding interactions as the adenine ring of ATP [18, 21, 22].

\section{Novel classes of gyrase inhibitors from natural sources}

In 2010 and 2011, two novel natural topoisomerase inhibitors were discovered: amycolamicin [23, 24] and kibdelomycin [25, 26] (Fig. 1D). Amycolamicin was isolated from a soil actinomycete, Amycolatopsis sp., and it has potent antibacterial activity against Gram-positive and some Gramnegative bacteria. Kibdelomycin was isolated from bacteria of the genus Kibdelosporangium, and it showed broad-spectrum, Gram-positive antibacterial activity. The structures of both amycolamicin and kibdelomycin include dichloropyrrole carboxylic acid, and they are both dual DNA GyrB and topoisomerase IV inhibitors. For kibdelomycin, the binding mode for both of these targets have been resolved, through the co-crystal structure of kibdelomycin with the 24-kDa fragment of S. aureus GyrB (PDB: 4URM) and with S. aureus ParE (PDB: 4URL). These studies showed a novel U-shaped binding mode, and several derivatives of kibdelomycin and kibdelomycin A were prepared [27].

An interesting recent follow-up to the binding mechanism of the classical aminocoumarins was the discovery of the bifunctional antibiotic simocyclinone, which is produced by Streptomyces spp. [19] The structure of simocyclinone D8 (Fig. 1E) differs from other aminocoumarins because of the absence of a deoxysugar moiety, and thus it cannot bind to the 'classical' binding site and act as a competitive inhibitor of GyrB, which might have been expected. X-ray crystallographic studies have shown that simocyclinone D8 binds with the aminocoumarine and angucyclinone moieties via two different pockets of the GyrA subunit (PDB: 2Y3P) [28].

Compounds from natural sources often have excellent in-vitro antibacterial activities, but they are often not suitable for clinical use due to their high molecular weights, and in many cases, their lack of drug-like properties and non-optimal bioavailability. However, they can serve as promising leads for further optimisation [22, 29]. These undesired properties of natural compounds can be overcome through the design of synthetic inhibitors with further drug-like properties. 


\section{Novel synthetic classes of gyrase inhibitors}

From the synthetic approach, several new structural classes of inhibitors of GyrB and ParE have been reported in more recent years, which have included benzimidazoles [30], pyrrolamides [31-34], pyrrolopyrimidines [35, 36], pyridylureas [37] and pyrazolopyridones [38, 39]. As illustrated in Figure 2, all of these classes of compounds have a common motif that can form a H-bond donor-acceptor network with Asp73 of E. coli GyrB [40]. They also include an aromatic moiety that can form $\pi$-cation interactions with arginine residues [38].

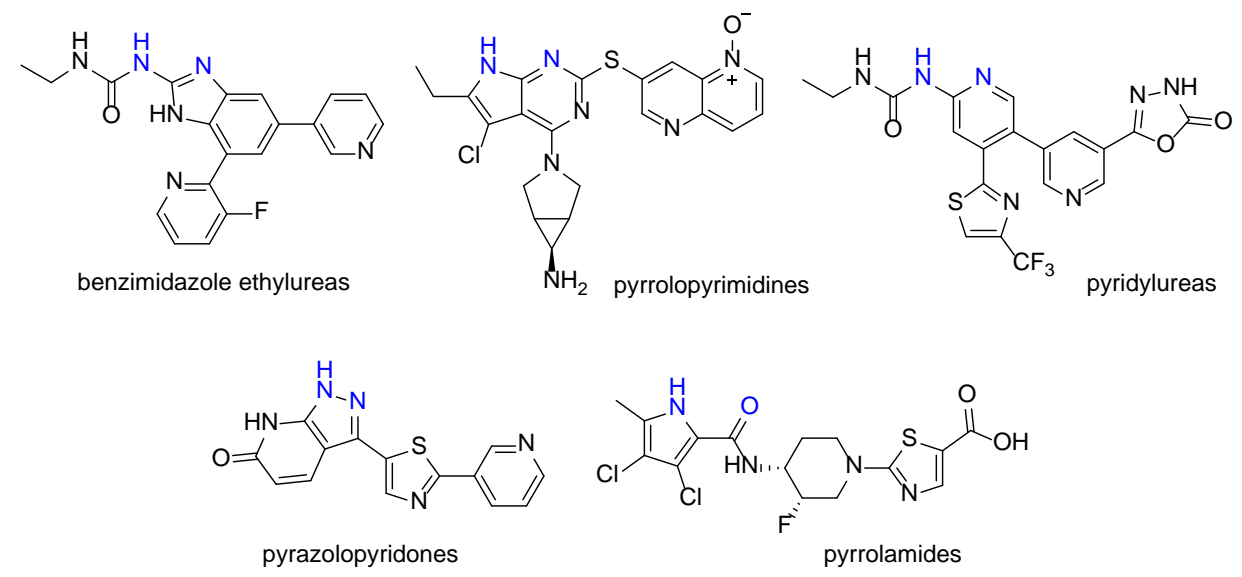

Figure 2. Structures of some of the more recent GyrB inhibitors, with the H-bond donor-acceptor motif highlighted [30-35, 37-40].

For readers interested in a broader and historical overview of GyrB inhibitor discovery, we recommend the review of Bisacchi and Manchester that covers the 50 years of effort towards ATPase inhibitors of DNA gyrase and topoisomerase IV [16], along with the review of Meyer [41]. For strategies relating to how to counter mechanisms of resistance by targeting bacterial topoisomerases, we suggest the review of Tse-Dinh that covers GyrA and GyrB [42]. For readers interested in developing antituberculotic agents through targeting topoisomerases from Mycobacterium tuberculosis, we recommend the recent review of Nagaraja [43]. In the current review, we will focus on the last 3 years in the discovery of inhibitors of the ATPase activity of DNA GyrB (and ATPase inhibitors of DNA topoisomerase IV, where appropriate). These aspects include the strategies and accomplishments regarding the new chemistry, the improvements to enzyme affinities, and the achieving of better antibacterial activities and more favourable pharmacokinetic properties.

\section{New scaffolds}

\section{Fragment-based screening leading to nitrogen heterocycles}

The search for novel structural classes of GyrB inhibitors and their further optimization is one of the main medicinal chemistry approaches to overcome the existing problems of antibacterial resistance. 
Several new scaffolds that have been obtained through different strategies, be they classical or novel, have been reported recently.

Structure-guided design of a pyrazolopyridone hit that was obtained from nuclear magnetic resonance (NMR)-based fragment screening led Cross et al. [38, 39] to (pyridin-3-yl)thiazolepyrazolopyridone $\mathbf{1}$. The co-crystal structure of $\mathbf{1}$ with the 24-kDa fragment of S. aureus GyrB (PDB: 5CTY) demonstrated the H-bond network between pyrazole, Asp81 (as $S$. aureus numbering) and a conserved water molecule. As in the azaindole scaffolds, the pyridine ring formed similar $\pi$-cation interactions with Arg84, and H-bonds with Arg144. The pyridone carbonyl interacted with Asn54, which is believed to be an essential interaction for improved potency of tricyclic inhibitors, as reported previously by $\mathrm{Li}$ and colleagues $[44,45]$.

One of the strategies was to optimise the water network in the binding site. Water analysis showed that water molecules were highly bound to Asp81, Gly85 and Thr173 at the site of the pyridine nitrogen, which suggested the demanding possibility of their displacement without any very suitable alternative interactions. On the other hand, poorly bound water molecules were predicted to overlap with the pyrazolopyridone moiety in the hydrophobic pocket. Further structure-activity relationship (SAR) studies were based on solvent analysis, which showed that water molecules in the hydrophobic pocket are still bound to some extent even in the presence of inhibitors. Displacing these molecules using a shorter alkyl chain on the inhibitors provided good space to manoeuvre to improve the binding affinity. Indeed, substitutions on the nitrogen of the pyridone ring showed a trend that was dependant on the length of the alkyl substituent: the longer the chain, the greater the improvement to both enzymatic and cell activity due to the better fit of the alkyl chain into the hydrophobic pocket. The N-propyl group then completely displaced the water molecule and provided the optimal balance to the desired properties ( $\mathrm{IC}_{50}, 0.006 \mu \mathrm{M}$ vs. S. aureus GyrB; MIC, $0.06 \mu \mathrm{g} / \mathrm{mL}$ vs. methicillin-sensitive S. aureus). Here, hydrophilic substituents were not successful at all. At the same time, substitutions in other positions of the pyridone ring (Fig. 3A, general structure I) showed various requirements, whereby only small substituents were tolerated in position $\mathrm{R}^{2}$, and position $\mathrm{R}^{3}$ could only be hydrogen due to the need to maintain the small dihedral angle between the thiazole and pyrazolopyridone rings. Substitutions on position 2 of the pyridine ring were used to explore the physico-chemical properties, and again, small substituents showed the best activities. Derivatives that lacked the pyridyl ring lost significant activity, which supported the previously suspected importance of the Arg84 and Arg144 interactions.

Characterisation of $S$. aureus ParE ATPase inhibitory activity was carried out for derivatives 2 and 3 (Fig. 3A). Both showed activity against the ParE subunit, although they were much weaker than against GyrB. When tested against a ciprofloxacin-resistant strain of methicillin-resistant S. aureus (MRSA), both compounds maintained their MIC, which suggested a reduced possibility of target-based fluoroquinolone resistance. Moreover, both of these compounds showed activity against other Grampositive pathogens, thus indicating their broad spectrum of action. 


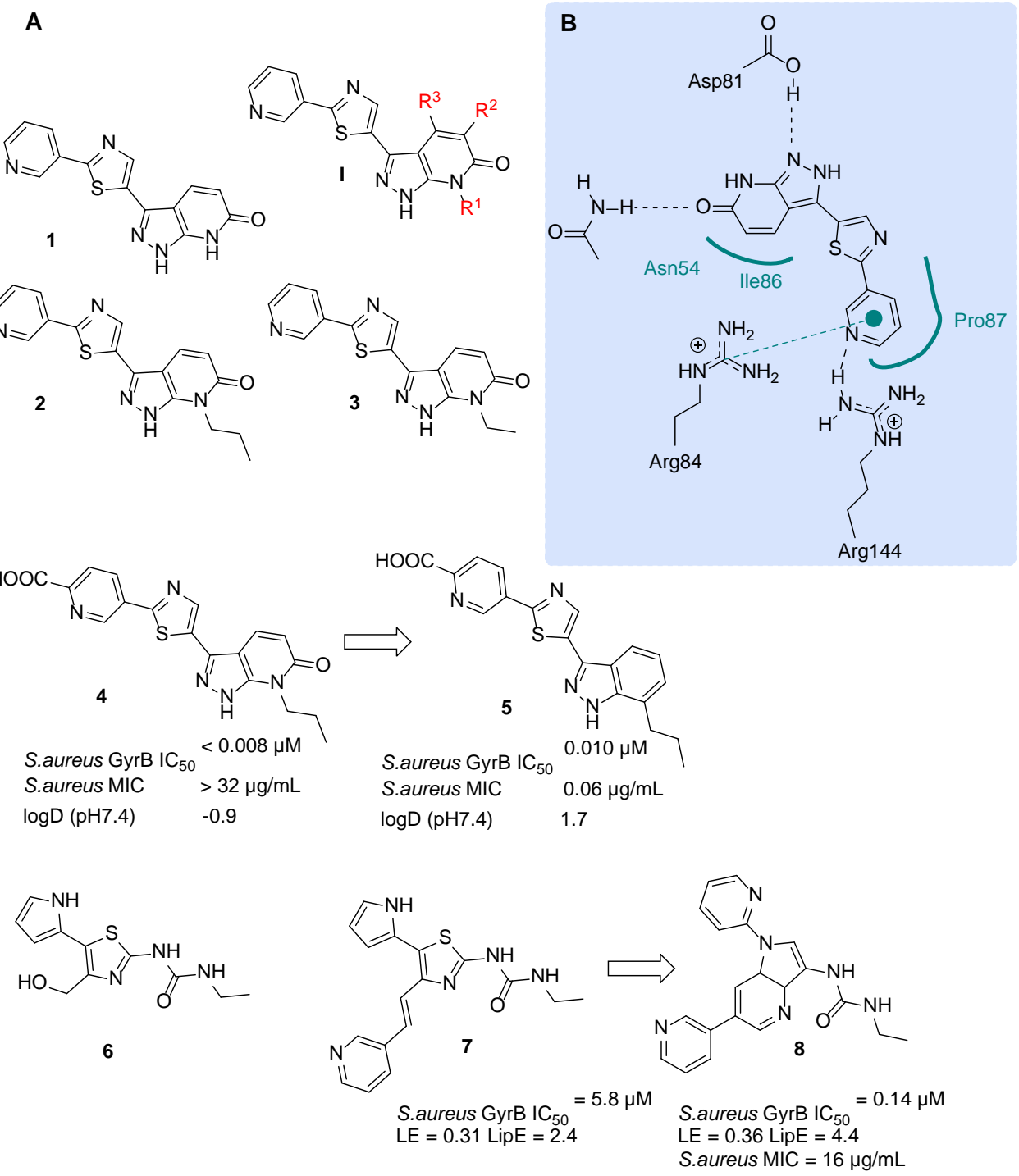

Figure 3. (A) Structures of various pyrazolopyridones, thiazole ureas and azaindoles. (B) Interaction scheme of pyrazolopyridone inhibitor 1 and the 24-kDa fragment of S. aureus GyrB (PDB: 5CTY) [39, 46-48].

Further optimization using fragment-based screening and scaffold hopping resulted in pyrazolopyridone analogue 4 , which contained a carboxylic acid functional group and showed $\mathrm{IC}_{50}<8$ $\mathrm{nM}$ against $S$. aureus GyrB [46]. Its good enzymatic activity was supported by the $\mathrm{X}$-ray crystal structure of the analogous pyrazolopyridone $\mathbf{2}$ (which lacks the carboxylic group) with the 24-kDa GyrB domain from S. aureus (PDB: 5D7D; Fig. 4A). Derivative 4 showed important interactions with the ATP binding pocket, which included the H-bond donor-acceptor network with Asp81, $\pi$-cation interactions of the pyridine ring and Arg84, and H-bonds with Arg144 (when the carboxylic acid moiety was exposed to a water environment). However, this carboxylic acid $\mathbf{4}$ showed only weak antibacterial activity against $S$. aureus (MIC, $>32 \mu \mathrm{g} / \mathrm{mL}$ ), which was possibly due to poor membrane penetration because of its polar scaffold, and hence a low logD. Increasing logD to improve cell penetration was shown to be a promising 
strategy, and the antibacterial results for Gram-positive strains were significantly lower for indazole $\mathbf{5}$, as shown in Figure 3.

The focus on exploration of the physicochemical properties and binding affinities of the heteroaryl moiety led to further optimization. Although several substituents showed increased solubility and improved physico-chemical properties in general, it turned out that the original carboxypyridine moiety was actually optimal. Structural rigidity and the H-bond interaction with Arg144 were crucial for excellent enzymatic and antibacterial activity against Gram-positive pathogens.



Figure 4. (A) X-ray crystal structure of 2 in complex with the 24-kDa GyrB domain (PDB: 5D7D) [47]. (B) X-ray crystal structure of 7 with the 24-kDa fragment of S. aureus GyrB (PDB: 5D6Q). Prepared using PyMol [49].

In the continuing studies of Zhang et al. [47], structure-based de-novo virtual design strategies were used to obtain aminothiazole $\mathbf{6}$ as a starting point for further SAR studies. The solving of the crystal structure of the 24-kDa fragment of $S$. aureus GyrB in complex with 6 (PDB: 5D6P) revealed important interactions among the thiazolyl urea, Asp81 and a conserved water molecule, with the ethyl group fitting into a small lipophilic pocket; more importantly, there were no interactions with Arg84 or Arg144, which had been regarded as essential for good potency of these inhibitors [48]. Based on those findings, and together with the desire to reach two Arg residues, pyridine derivative $\mathbf{7}$ was synthesized. The new crystal structure (PDB: 5D6Q) showed that the pyridine ring interacted with Arg84 and Arg144 via $\mathrm{H}$-bonds and $\pi$-cation interactions, as well as the thiazole and urea moieties, creating a H-bond network through a conserved water molecule, as previously seen. However, another interaction of a nonconserved water molecule and the thiazole nitrogen near Asp81 was noted, and this provided the inspiration to displace the non-conserved water molecule with a heteroatom (8) to increase the binding affinity. The co-crystal structure of 8 with the 24-kDa fragment of S. aureus GyrB (PDB: 5D7C) demonstrated this displacement of the non-conserved water molecule with the pyridine nitrogen, which together with the $\mathrm{NH}$ of urea, formed an additional H-bond network with Asp81. Inhibitor $\mathbf{8}$ showed the improved $\mathrm{IC}_{50}$ of $0.14 \mu \mathrm{M}$ against $S$. aureus GyrB, and a MIC of $16 \mu \mathrm{g} / \mathrm{mL}$ against an MRSA strain, and was then used for further SAR studies on the azaindole scaffold. Azaindole N1 aryl substitutions 
revealed the importance of the dihedral angle between the moieties and the azaindole core. Replacement of the 2-pyridyl moiety of $\mathbf{8}$ with 2-pyrimidyl released the torsional stress, and indeed showed improved $\mathrm{IC}_{50}$ and MIC, with the further studies focused on 2-pyrimidyl derivatives. To improve the physicochemical properties, SARs for non-aryl substitutions were carried out, with most of these enzymatic activities in the micromolar range. After optimization of the N1 substitution, additional studies of C6 substituents were carried out to improve the aryl interactions with the arginine residues, and to also improve the physicochemical properties of these compounds. In comparison with 2-pyridyl of starting structure 8, the pyridone moiety showed advanced interactions with arginine residues and good cell penetration. Combination of these optimal substitutions resulted in a series of potent de-novo GyrB inhibitors that showed improved activities as well as improved MIC profiles against Grampositive pathogens.

\section{Pyrrolamides and their analogues}

A library of compounds inspired by the natural marine compound oroidin [50] was screened for GyrB inhibitory activity, which defined cyclised derivative $\mathbf{9}$ as the best hit for $E$. coli inhibitory activity ( $\mathrm{IC}_{50}$, $12 \mu \mathrm{M})$ [51]. Introduction of a oxalyl moiety improved the GyrB inhibitory activity by 20-fold compared to the amino analogue, giving the 5,6,7,8-tetrahydrobenzothiazole derivative $\mathbf{1 0}$ with an $E$. coli $\mathrm{GyrB}$ $\mathrm{IC}_{50}$ of $58 \mathrm{nM}$. Docking studies suggested that the pyrrole nitrogen formed a H-bond with Asp73 (as E. coli numbering) and that a H-bond network was established between the pyrrolamide oxygen, the Asp73 side chain, and a highly conserved structural water. Indeed, pyrroles are commonly present motifs in GyrB inhibitors, as the 5-methylpyrrole moiety is found in chlorobiocin, and the 5-methyl-3,4dichloropyrrole moiety is found in amycolamicin and kibdelomycin, and also in a series of synthetic 5methyl-3,4-dichloropyrrole derivatives from Astra Zeneca that showed GyrB inhibition [31, 52, 53]. Introduction of a carboxylic moiety was beneficial, through interactions with Arg136. These compounds showed only modest antibacterial activities against Gram-positive bacterial strains, while their evaluation against wild-type, impA and $\triangle$ tolC E. coli strains suggested that they are efflux pump substrates and/or do not have the physicochemical properties necessary for cell-wall penetration. 
oroidin<smiles>Nc1nc2c(s1)CC(NC(=O)c1cc(Br)c(Br)[nH]1)CC2</smiles>

$\mathrm{IC}_{50}=\stackrel{9}{12 \mu \mathrm{M}}$

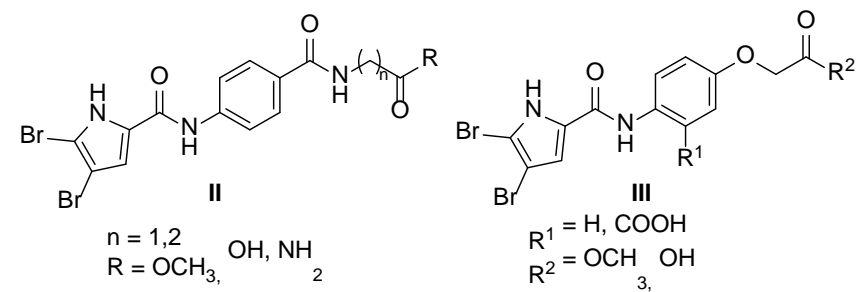
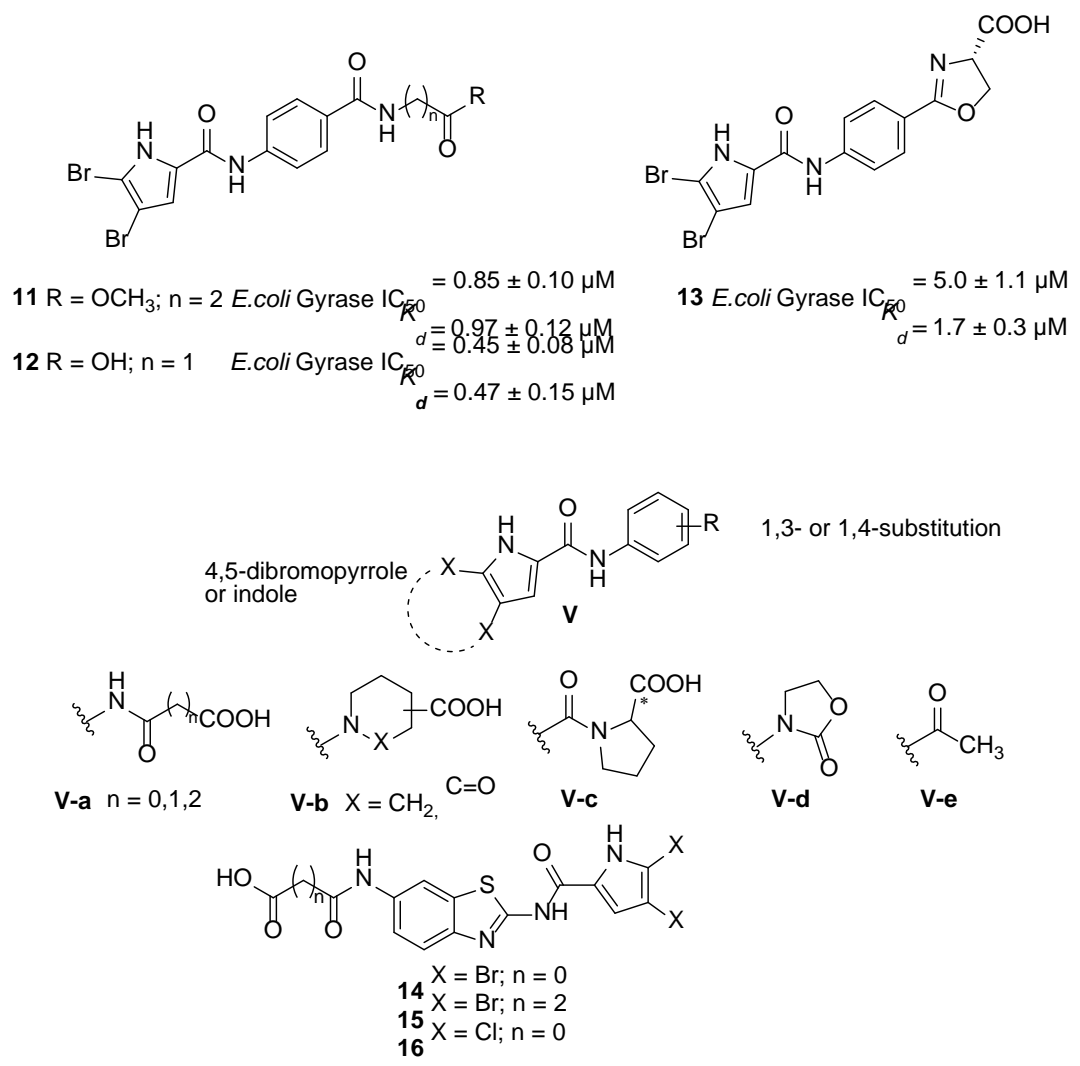

Figure 5. Structures of the pyrrolamide-based inhibitors [32, 33, 51, 54].

Zidar and colleagues [33] continued their studies with the preparation of a novel class of $\mathrm{N}$ phenyl-4,5-dibromopyrrolamides and $N$-phenylindolamides as GyrB inhibitors, through the modification of the 5,6,7,8-tetrahydrobenzothiazole series (Fig. 5). The 4,5-dibromopyrrolamide and indolamide moieties were incorporated to establish the crucial H-bond donor-acceptor network. Optimal interactions with Arg76 and Arg136 were searched for using different substituents on the 4position of aniline, along with changing the length of the linker chain. As well as the free carboxylic acid derivatives, the corresponding methyl esters and carboxamides were also prepared to demonstrate the important role of the acidic functional group for activity. It was not possible to determine the 
importance of the linker length, although carboxylic acids were generally more active than the corresponding esters and the primary carboxamides. However, particular carboxamides were shown to have improved antibacterial potential, apparently due to better cell-wall penetration. Several compounds showed $\mathrm{IC}_{50}<1 \mu \mathrm{M}$. Selected potent compounds were also tested against $S$. aureus gyrase and topoisomerase IV of both E. coli and S. aureus strains, and these showed weaker activities than for $E$. coli GyrB. This was interpreted to be the consequence of the small structural changes in the binding site of these enzymes, as the GyrB adenine-binding pocket of S. aureus is smaller than that of E. coli. Similarly, replacement of Ile78 in GyrB to Met74 in ParE resulted in partial conformational changes that might lead to different binding effects $[30,55]$. The crystal structure of the 43-kDa GyrB domain of E. coli and 12 (PDB: 4ZVI) was solved, which confirmed the binding mode of this ligand (Fig. 6A). H-bonds were also seen between Asp73 and the pyrrole nitrogen, and between Arg76 and the carbonyl. The flexible carboxylic group was in a position with weak electron density, and therefore might not have been in contact with Arg136, as the molecular docking suggested. Molecular dynamics simulation was thus carried out, and this showed that the distance between the Arg136 side chain and the carboxylate group of the ligand $\mathbf{1 2}$ was $2.8 \AA$ to $15.5 \AA$. The carboxylate group was in contact with the arginine side chain for $34 \%$ of the simulation time, and the possibility of an interaction was shown. However, flexibility of the carboxylate tail was confirmed as well, which explained the weak electron density seen in the crystal structure.

Based on the crystal structure obtained here, further studies looked to increase the inhibitory and antibacterial activities [32, 56]. The central core was changed to aminobenzene and the benzoyl scaffold, to provide hydrophobic interactions with Gly77, Ile78 and Pro79, and to support the possibility of other important interactions. The pyrrolamide or indolamide moieties were retained, as both have $\mathrm{NH}$ and a carbonyl group to form the crucial network with Asp73 and a conserved water molecule, as well as their indirect contact with Asn46. As an interaction with the arginine was hard to achieve, 28 structures were prepared to additionally determine the optimal length, position (i.e., para or meta substitution, stereochemistry) and rigidity of the carboxylic moiety. The oxazolidin-2-one and acetophenone derivatives were shorter and were not ionized, and thus they would be expected to show improved membrane penetration. The carboxylic acids were more potent than the corresponding esters, and the 1,4-substitution, rather than the 1,3-substitution, showed a more suitable conformation for the formation of the important interactions. The best activity was an $\mathrm{IC}_{50}$ of $0.18 \mu \mathrm{M}$, which was obtained for the 4,5-dibromopyrrol-2-yl derivative with an oxalyl moiety (Fig. 5. general structure $\mathbf{V}$-a). However, no significant antibacterial activities against Gram-positive and Gram-negative strains were obtained, neither for the most potent compound in the enzymatic assay, nor for its less acidic analogues; this was suggested to be an issue of cell entry and/or efflux pumps. Thus, these compounds did not show any improvements over previous series, and further demonstrated that the physico-chemical properties need to be optimised to achieve antibacterial activity. 
The continuation of these studies on the tetrahydrobenzothiazole scaffold-based GyrB inhibitors resulted in the original 4,5,6,7-tetrahydrobenzothiazole scaffold being converted to aromatic benzothiazole, with the intention to promote the formation of the $\pi$-cation interaction with Arg76 that is important for increased activity [54]. Two series of compounds with a benzothiazole scaffold and pyrrole-2-carboxamide substitutions on positions 2 or 6 were investigated. Moreover, the 4,5dibromopyrrole-2-carboxamide was replaced with a smaller 4,5-dichloropyrrole moiety, to potentially achieve activity also against $S$. aureus gyrase and E. coli and S. aureus topoisomerase IV, on the basis of the smaller volume of their hydrophobic pocket. SARs on series A of these compounds, where the pyrrole-2-carboxamide moiety was on position 6 of the benzothiazole, showed that an oxalyl moiety was the most potent, and that dibromo substitution of the pyrrole was better than the dichloro substitution. The best inhibitors showed inhibitory activities in the low nanomolar range, with the $E$. coli gyrase $\mathrm{IC}_{50}$ of $38 \mathrm{nM}$ for the oxalyl derivative, and $57 \mathrm{nM}$ for the succinyl derivative. Significant $S$. aureus gyrase inhibition was not achieved with these series A compounds. Series B had the pyrrole-2carboxamide moiety attached in position 2 of the benzothiazole, and showed similar advantage of the dibromo-substituted over the dichloro-substituted moieties. The best potency was achieved as for series A, with the dibromo-substitution and the oxalyl (14; IC $50,58 \mathrm{nM})$ and succinyl $(\mathbf{1 5}$; IC $50,33 \mathrm{nM})$ moieties. More pronounced differences between these two series were seen when they were tested on other strains and on topoisomerase IV. Series B showed better activities against $S$. aureus gyrase, with the best $\mathrm{IC}_{50}$ of $510 \mathrm{nM}$ for $S$. aureus GyrB inhibition. Additional assays for topoisomerase IV of both E. coli and S. aureus showed low micromolar activities of these carboxylic acids that were comparable or better than for novobiocin, while the corresponding esters were not active. This defined the importance of the carboxylic acid moiety. Some derivatives showed balanced activities against all four of these enzymes, and dichloropyrrole $\mathbf{1 6}$ had the best dual inhibition potential, with activities in the nanomolar or low micromolar range ( $\mathrm{IC}_{50}, 87 \mathrm{nM}$, for E. coli $\mathrm{GyrB}$ ). Antibacterial testing against two Gram-negative and two Gram-positive strains did not show any activity of note. Selected compounds were thus tested against $\triangle t o l C E$. coli, which has a non-functional efflux pump. With the malonyl derivative showing an activity with MIC $<16 \mu \mathrm{g} / \mathrm{mL}$, this suggested efflux pump issues of the similar derivatives. The crystal structure of $\mathbf{1 4}$ in complex with the 43-kDa domain of E. coli GyrB has been solved (PDB: 5L3J), with well-defined electron density of the ligand, with the exception of the flexible carboxylate group, as observed in the previous crystal structure (PDB: 4ZVI). The binding mode was confirmed as expected, with 4,5-dibromopyrrole forming the H-bond/ water network (Fig. 6). 


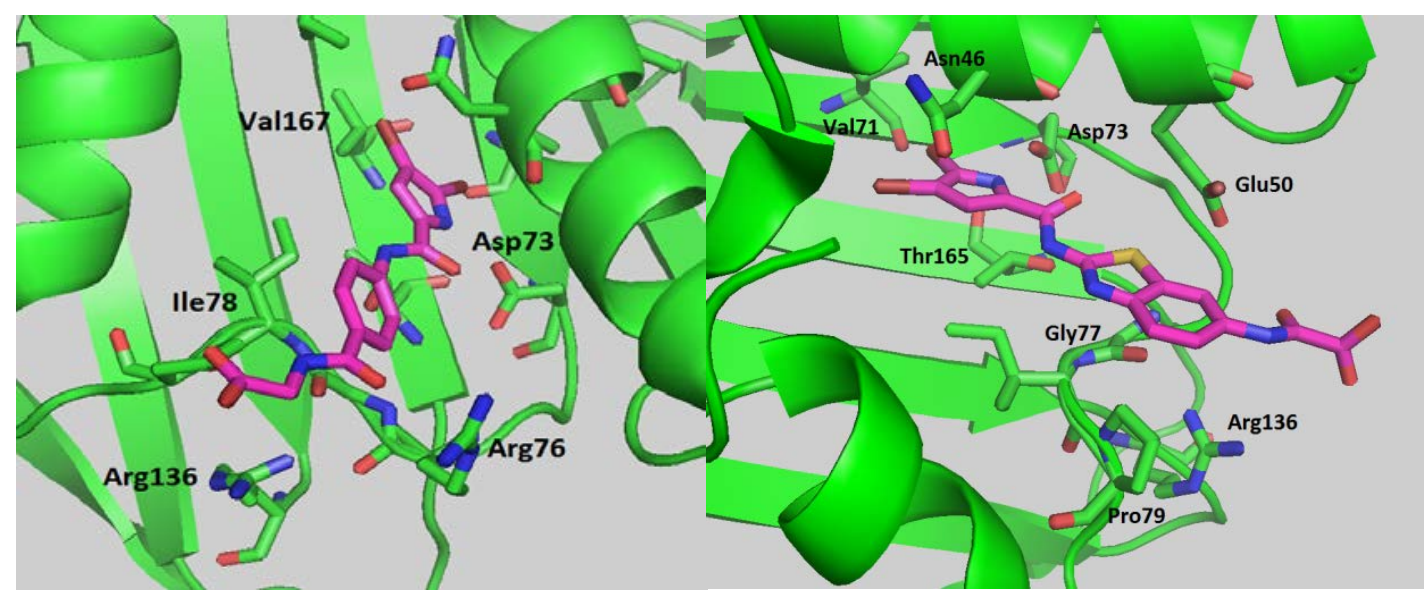

Figure 6. Crystal structures of the 43-kDa domain of E. coli GyrB in complex with 12 (PDB: 4ZVI) (A) [33] and 14 (PDB: 5L3J) (B) [54]. Prepared using PyMol [49].

Computational methods were combined with molecular dynamic simulations in a linker-switch approach with a saturated central scaffold to find new ATP binding site inhibitors of DNA GyrB [57]. Several derivatives based on the piperazin and piperidin 1,4-disubstituted linkers were prepared that kept the indole and pyrrole moieties, together with the carboxylic moiety (Fig. 7). However, these compounds showed weaker activities than their unsaturated analogues for E. coli GyrB ( IC $_{50}, 3.2 \mu \mathrm{M}$ for the best 4,5-dibromopyrrole piperidin-4-yl derivative), and they did not show any promising antibacterial activities.
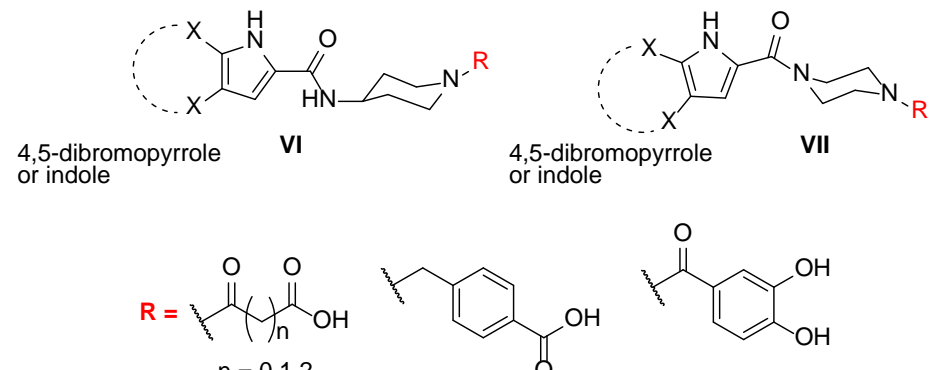<smiles>CC(C)(C)Cc1ccc(C(=O)O)cc1</smiles><smiles>CC(C)C(=O)c1ccc(O)c(O)c1</smiles><smiles>[R]C(=O)NC1CCc2nc(NC(=O)c3cc(Br)c(Br)[nH]3)sc2C1</smiles><smiles>[R]NC(=O)Cc1csc(NC(=O)c2cc(Br)c(Br)[nH]2)n1</smiles><smiles></smiles>

Figure 7. Structures of the pyrroleamide-based inhibitors [34, 57, 58]. 
Modification of the central scaffold was also a strategy that was investigated by Tomašič et al. [59], which provided a new series of 4,5,6,7-tetrahydrobenzo[1,2- $d$ ]thiazole-2,6-diamine derivatives with the 4,5-dibromopyrrole moiety in position 2 (Fig. 7, VIII) [34]. Moreover, a series based on the opened 2-(2-aminothiazol-4-yl)acetic acid ring (Fig. 7, IX) was synthesized, as the smaller flexible core allowed the introduction of larger substituents that could provide interactions with Arg76 and Arg136. In general, the enzymatic activities of the 4,5,6,7-tetrahydrobenzothiazole series were weaker in comparison with the analogues with the pyrrole moiety in position 6 , with the most potent inhibitor showing an $\mathrm{IC}_{50}$ of $0.89 \mu \mathrm{M}$. None of the inhibitors showed antibacterial activity against selected strains. The opened analogues were in general weaker inhibitors (best $\mathrm{IC}_{50}, 16 \mu \mathrm{M}$ ), although docking studies proposed that their binding was similar compared to the corresponding 4,5,6,7-tetrahydrobenzothiazole analogues. More lipophilic and rigid aromatic moieties with acidic substituents were thus introduced, including sulfonamide, phenol and benzoic and salicylic acids, but these showed weak activity (GyrB inhibitory activity for the best of these: $\mathrm{IC}_{50}, 33 \mu \mathrm{M}$ ). However, the enzymatic activities were also still too low to provide any antibacterial activities. When the pyrrole moiety was substituted with ethylurea, which is frequently found in other GyrB inhibitors, this resulted in less potent compounds with no antibacterial activities [59].

Another strategy for the modification was used by Frlan et al. [58], where the amino-acid tyrosine was used as the central scaffold. The 4,5-dibromopyrrole was maintained, and various aromatic, aliphatic acid and phenyl moieties were modified in the search for good activities (Fig. 7, general structure $\mathbf{X}$ ). These compounds showed GyrB inhibitory activities in the low micromolar range, and three had modest antibacterial activities against both S. aureus and Enterococcus faecalis. When the antibacterial activities were compared to their corresponding enzymatic activities, there was no clear correlation between these, which suggested that there were additional mechanisms of antibacterial action involved.

Based on a pyrrolamide series of inhibitors [31], both ligand-based and structure-based approaches led to a small library of analogues with a 5-phenyl-1,2,4-oxadiazole core [60]. The design of two series of compounds (Fig. 8) was guided by molecular docking. Bioisosteric replacement of the thiazole carboxylic acid with heteroaromatic oxadiazole-carboxylic acid had a large role in the $\pi$ stacking with Arg76, as well as for H-bonds with Arg136. Different substitutions on the pyrrole moiety were made to explore the lipophilic pocket of GyrB. A methylene or imine bridge was inserted in the second series of compounds to better direct the carboxylic group towards Arg136. The imine hydrogen formed an additional H-bond with Gly101. Supercoiling assays demonstrated that the indolyl moiety in combination with the carboxylic acid were important in series A (E. coli gyrase: $\mathrm{IC}_{50}, 4 \mu \mathrm{M}$ ). This compound was not active against the other enzymes tested. On the other hand, the best inhibitor in series $\mathrm{B}$ was with a pyrrolecarboxamide moiety, while the indole derivatives were not active. The most potent inhibitors in series B were compounds with an imine bridge and 4,5-dibromopyrrole (IC $50,5.8 \mu \mathrm{M}$ ) and 3,4-dichloro-5-methylpyrrole ( $\mathrm{IC}_{50}, 1.2 \mu \mathrm{M}$ ) moieties. This second compound also showed balanced 
micromolar activity against $E$. coli topoisomerase IV and $S$. aureus gyrase and topoisomerase IV (IC 50 , 1.2-135 $\mu \mathrm{M})$. These data supported the role of the interaction between the imine hydrogen and Gly101. The best inhibitors were further tested for antibacterial activities against two Gram-positive and two Gram-negative strains; however, none of these showed any significant antibacterial activities.

A strategy of Daiichi Sankyo for compounds with low cytotoxicity, high water solubility, dual GyrB/ParE inhibition, and sufficient in-vitro antibacterial activity resulted in two novel classes of inhibitors with an imidazole carbonyl moiety [61]. Here, compounds with substituted imidazole as the left-hand substituent in Figure 8 and with a hydrocarbon or heteroaromatic linker (Fig. 8, general structure XIV) met all of these desired criteria. Continued improvement here lead to the discovery of 11 derivatives of 2-hydroxyalkyl-1,3,4-thiadiazole [62] (Fig. 8, general structure XV). A hydroxyalkyl substitution $\left(\mathrm{R}^{2}\right)$ improved the properties here in comparison to the previous analogues. The better solubility (and thus better potential bioavailability), in-vitro antibacterial activity, lack of cytotoxicity, and efficacy and safety of these compounds might result in inhibitors of antibiotic-resistant pathogens that are suitable for human use, currently is compound (DS-2969b) in early development for the treatment of $C$. difficile infections.

A
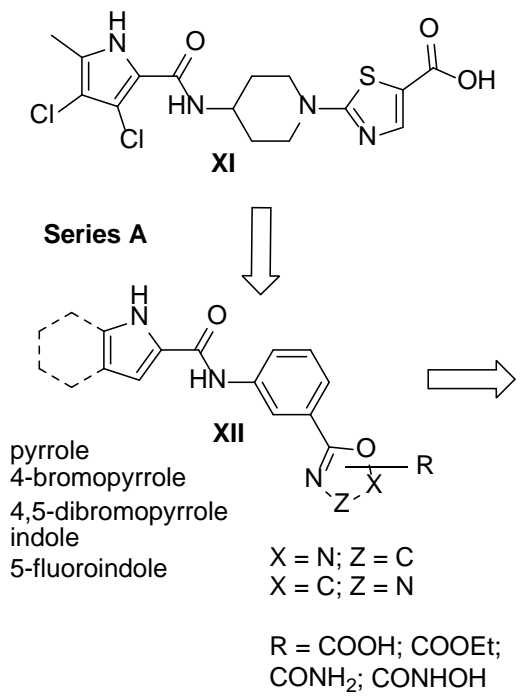

\section{B}

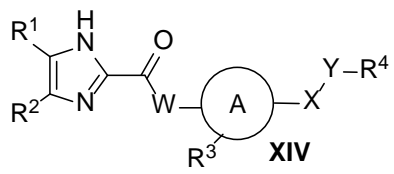

Series B
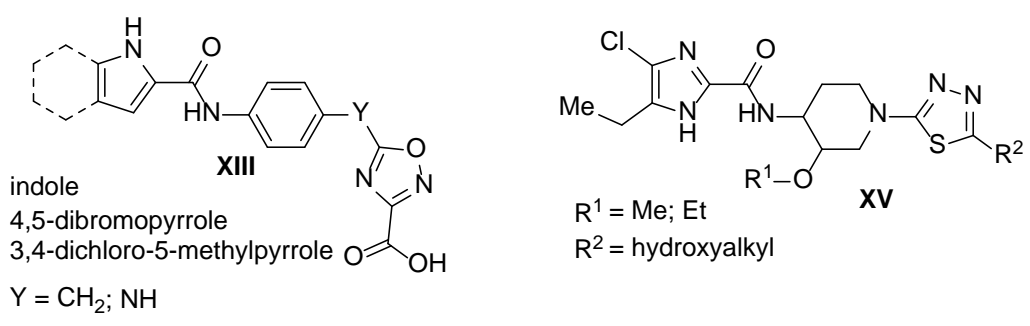

Figure 8. (A) Structures of inhibitors with the 5-phenyl-1,2,4-oxadiazole core [60]. (B) Structures of inhibitors with the imidazole moiety [61, 62].

\section{Modern techniques in scaffold search and optimization}

Fragment-based screening represents another strategy for successful drug discovery [63, 64]. This has allowed the discovery of small chemically diverse fragments, with the description of their binding modes. Suitable weak compounds can then be optimized through structure-based design. This strategy 
was exploited by Mesleh et al.. from Cubist Pharmaceuticals [38], who used different bio-physical techniques to obtain efficient GyrB lead ligands. First, using saturation-transfer difference (STD)-NMR spectroscopy, they narrowed down thousands of fragments into 300 hits that were classified according to their spectra signal intensity and novelty. Next, 46 fragments were selected and characterized using ligand-observed binding measurements with STD-NMR, against the 73-kDa S. aureus GyrB and the 43kDa domain of E. coli ParE. Additionally, chemical shift perturbation via protein-observed ${ }^{1} \mathrm{H}-{ }^{15} \mathrm{~N}$ heteronuclear single quantum coherence NMR measurements were used to determine the contributions of the binding site. For three hit compounds (PDB: 5CPH, 5CTU, 5CTW) and two optimized fragments (PDB: 5CTX, 5CTY; Fig. 10), the X-ray crystal structures with S. aureus GyrB were solved at a resolution of $<2 \AA$.
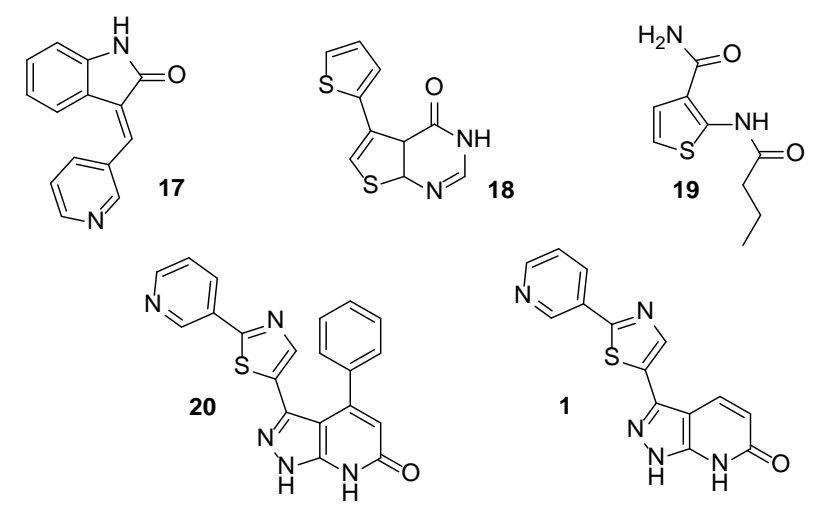

Figure 9. Structures of the initial indolinone 17 (PDB entry 5CPH), thienopyrimidinone 18 (5CTU) and thiofen 19 (5CTW) fragments, and of the optimized pyrazolopyridines 20 (5CTX) and 1 (5CTY) $[38,39]$.

The binding of indolinone $\mathbf{1 7}$ was not optimal. The pyridyl ring was located close to Ile86 and Ile102 instead of forming the crucial interactions with Arg84 and Arg144, which resulted in little binding to the 73-kDa S. aureus GyrB, and suggesting possible steric issues for the flexible loop. This scaffold was abandoned due to the unsuccessful changes in the position of the pyridyl ring, with problematic E/Z isomerization. The geometry and positioning of thienopyrimidinone $\mathbf{1 8}$ was not optimal either, due to many conformational issues, and optimization was not even feasible. There were similar issues with the thiophen scaffold 19.

Structures $\mathbf{2 0}$ and $\mathbf{1}$ are analogues of the first-obtained fragments. The study of the co-crystal structure of $\mathbf{2 0}$ demonstrated the important role of both the pyrazol moiety and the carbonyl group, and the beneficial position of the pyridine ring, which managed interactions with Arg84 and Arg144 due to the thiazole ring. Further conformational analyses suggested better outcomes for compound 1, so with the phenyl ring removed, and thus with a more favourable torsional angle of $22^{\circ}$. The activity showed five-fold improvement ( $\mathrm{IC}_{50}, 0.26 \mu \mathrm{M}$; ligand efficiency, 0.44), and supported these theories. Overall, the study of crystal structures from fragment screening together with torsional angle analysis have 
proven to be a successful strategy for fragment-based discovery, and promising compounds have been provided for further optimization.

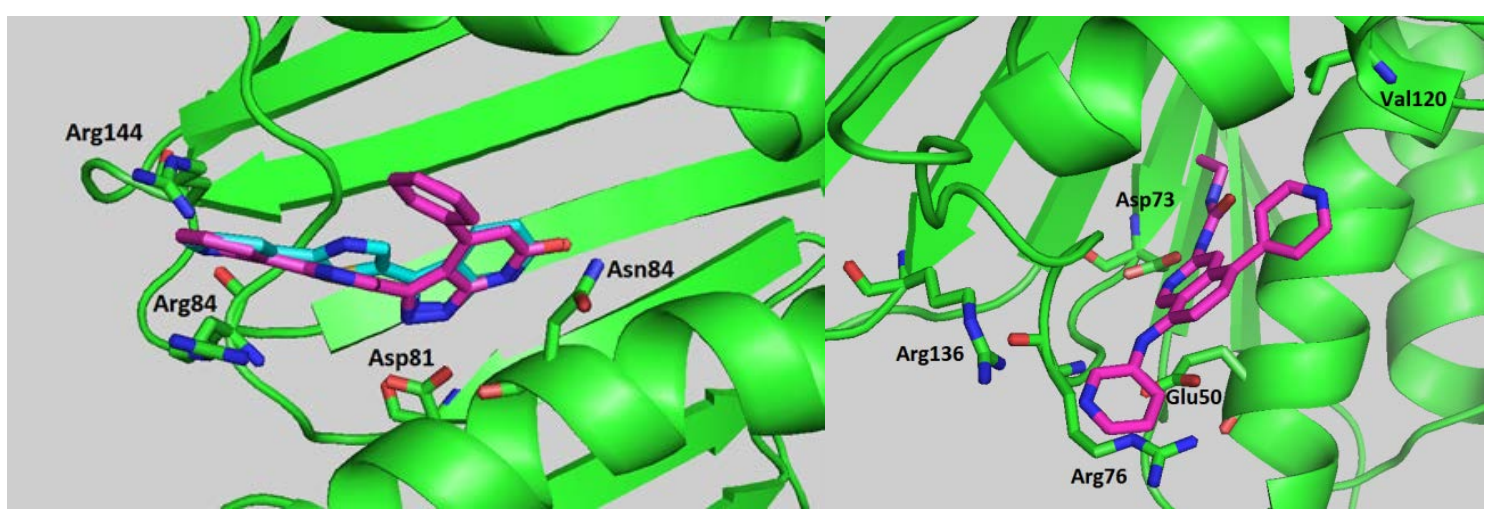

Figure 10. Interactions of pyrazolopyridines 20 and 1 with the 73-kDa S. aureus GyrB (5CTY, 5CTX) [38, 39]. Prepared using PyMol [49].

Panchaud et al. [65] carried out hit-to-lead programmes and lead optimization, with a focus on active inhibitors against Gram-positive and respiratory tract pathogens. They also previously reported on their optimized H-bond networks of ethylureas [66] (i.e., formation of two H-bonds with Asp73) that was used as a starting point. The screening of different scaffolds with the mutual ethylurea motif led to the identification of 1-ethyl-3-isoquinolinyl urea as the most promising scaffold (IC $_{50}, 20 \mu \mathrm{M}$; ligand efficiency, 0.66), which was supported by molecular modelling (Fig. 11A, XVI). Scaffold XVI was used for a further fragment evolution programme, which showed that positions 5 and 8 were the most suitable for derivatization. Pyridyl substitution in position 5 led to the most active compound 21 (E. coli gyrase: IC $_{50}, 0.13 \mu \mathrm{M}$ ), which also showed good antibacterial activity against $S$. aureus (MIC, 0.5 $\mu \mathrm{g} / \mathrm{mL}$ ) and good oral efficacy. The crystal structure of 22 with the 24-kDa GyrB (PDB: 5MMN) showed the importance of the pyridyl substitution, with the formation of water-mediated H-bonds with the Val120 backbone nitrogen. Investigation of substitutions to position 8 (see Fig. 11A) resulted in compounds 23 (PDB: 5MMP; Fig. 11B) and 24 that showed improved enzymatic activities. However, their in-vivo oral efficacies were no better than for 22. Finally, carbamate substitution of position 8 was introduced, and these compounds showed good enzymatic and antibacterial activities (25; E. coli gyrase: $\mathrm{IC}_{50}, 0.03 \mu \mathrm{M}$; S. aureus: MIC, $0.06 \mu \mathrm{g} / \mathrm{mL}$ ), and also good oral efficacy. The crystal structure of one of the derivatives (PDB: 5MMO) showed a stacking interaction of the carbamate with Arg76, and an H-bond with Arg136. A panel of various pathogens was used to test these compounds for their antibacterial activities. The aminopyridyl substitution at position 8 showed improved activity against all of the bacteria tested (e.g., 21: S. aureus: MIC, $0.5 \mu \mathrm{g} / \mathrm{mL} v$ s. 24: MIC $\leq 0.03 \mu \mathrm{g} / \mathrm{mL}$ ). The carbamates only showed improvements over $\mathbf{2 1}$ against $S$. aureus and Streptococcus pyogenes. Here, efflux pump issues were observed for all of the compounds, as they were only weakly active against wild-type $E$. coli and showed better activity against the $\Delta t o l C$ knock-out mutant strain. When tested against various enzymes from different bacterial strains, including topoisomerase IV and fluoroquinolone-resistant 
gyrase, these compounds showed balanced activities. To increase the solubility of $\mathbf{2 5}$, the phosphate prodrug was made, with a phosphate group attached to the 4-pyridyl moiety. The efficacy of this prodrug in a neutropenic murine screening model was increased significantly. However, hERG K-channel block was observed for 25, such that increased lipophilicity caused stronger hERG block, while decreased $\mathrm{pK}_{a}$ improved the MIC. However, no further compounds showed balanced hERG block and antibacterial activity as well as in-vivo efficacy.

A


Figure 11. (A) Scheme of the fragment evolution programme. (B) Crystal structure of 23 and the 24kDa E. coli GyrB (PDB: 5MMP) [65]. Prepared using PyMol [49].

\section{Mycobacterium tuberculosis DNA gyrase}

\section{Inhibitors focused on Mycobacterium tuberculosis DNA gyrase}

Mycobacterium tuberculosis has DNA gyrase as its only representative of the type II topoisomerases, and thus has no topoisomerase IV [67]. Moreover, there are some differences in the gyrase structures of Gram-positive and Gram-negative strains. There is a contiguous sequence of 165 amino in the Cterminal domain of GyrB of Gram-negative bacteria, while this is lacking in Gram-positive bacteria, which might thus involve alternate DNA binding regions [68]. However, the motifs important for the ATPase activity of GyrB (i.e., Glu42, His38) are conserved across all bacteria, which ensures a similar reaction cycle. There is 63\% homology between GyrB of E. coli and M. tuberculosis [69], and the differences in their protein sequences might have some influence on the catalytic properties (i.e., supercoiling $v s$. decatenation) or the efficacy of the enzyme activity [70]. 
Reddy et al. [71] reported on two novel classes of mycobacterial GyrB inhibitors that were obtained through molecular hybridization. Based on their previous studies where they had defined the importance of hydrophobic interactions for mycobacterial GyrB specificity, they re-engineered their lead compound with two goals in mind: to increase the hydrophobicity, so as to improve the potency against GyrB; and to evaluate the steric and electronic effects, to promote the antimycobacterial activity. The benzofuran scaffold and the urea side chain were selected as the starting point for this molecular hybridization. Benzofuran was kept or changed for benzoisothiazole, different urea or thiourea substituents were used (see Fig. 12), and the aminopiperidine linker was replaced with piperazine. ATPase assays were carried out with Mycobacterium smegmatis, and these showed more than $75 \%$ inhibition at $200 \mu \mathrm{M}$ for all of these compounds. The most potent compound 26 had an $\mathrm{IC}_{50}(\mathrm{M}$. smegmatis) of $1.8 \mu \mathrm{M}$. Differential scanning fluorimetry was also carried out, which defined the stable protein-ligand complex with a positive shift of $3.5^{\circ} \mathrm{C}$.
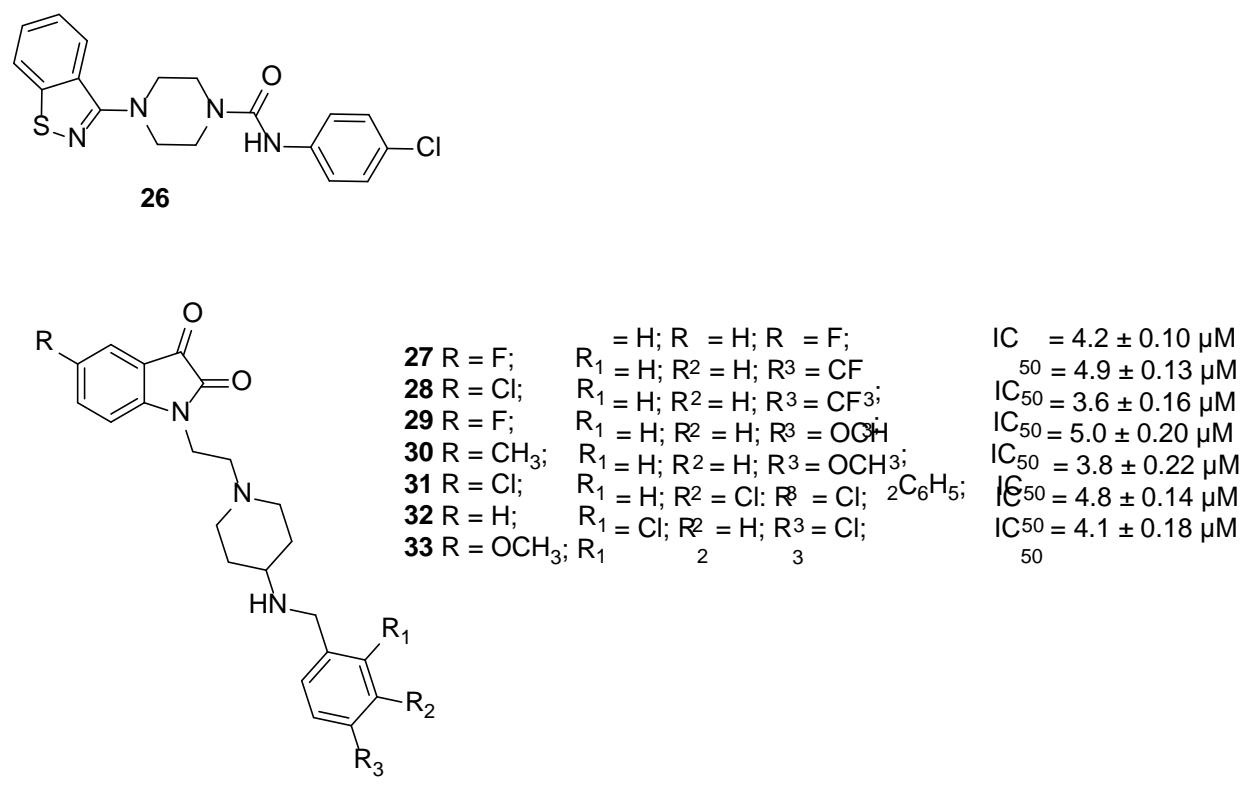

Figure 12. Structures of the most potent benzoisothiazole $\mathbf{2 6}$ reported by Reddy et al. [71], and structures and activities of the most potent indoline-2,3-dione derivatives [72].

Previous studies that used an $\mathrm{N}$-linked 4-aminopiperidine linker between the bicyclic and heteroaryl moieties [73-75] induced the re-engineering efforts of Jeankumar et al. [72]. They carried out molecular hybridization of the linker, indoline-2,3-dione, and the heteroaryl ring to explore the pharmacophoric criteria for activity against M. tuberculosis GyrB. The hydrophobic indoline-2,3-dione was included due to the known hydrophobic pocket in GyrB that supported the specificity of the enzyme. The heteroaryl moiety (see Fig, 12) that influenced the M. tuberculosis selectivity was chosen based on previous SAR studies.

In DNA supercoiling assays, eight of these compounds showed activities against M. tuberculosis DNA gyrase (Fig. 12; $\mathrm{IC}_{50},<5 \mu \mathrm{M}$ ) and selectivity against GyrB of S. aureus, E. coli or Pseudomonas 
aeruginosa. The compounds with activities against $M$. tuberculosis that were $<50 \mu \mathrm{M}$ were further screened in the M. smegmatis ATPase assay. The lower activities against $M$. tuberculosis were explained by the slow-growing mechanism, and $M$. smegmatis was considered to be a surrogate target. The most potent compound was $\mathbf{2 9}$, with a $M$. smegmatis $\mathrm{GyrB}_{\mathrm{I}} \mathrm{C}_{50}$ of $10.6 \mu \mathrm{M}$, and a M. tuberculosis gyrase supercoiling $\mathrm{IC}_{50}$ of $3.6 \mu \mathrm{M}$. Docking studies suggested important interactions between the aminopiperidine linker and Asp79 and Glu56. The trifluoromethylbenzyl moiety was deeply immersed in the hydrophobic pocket and formed hydrophobic interactions with Val49, Ile84 and Met100, while the ethyl bridge between the linker and the indoline-2,3-dione formed interactions with Pro85. Differential scanning fluorimetry confirmed the good activity of 29, with a positive shift that demonstrated the thermal stability of this protein-ligand complex ( $T_{m}$ shift, $\left.2.9{ }^{\circ} \mathrm{C}\right)$. The further screening for in-vitro antimycobacterial activity was mostly in agreement with the supercoiling assays, and this showed low micromolar activities for compounds 27, 29 and 31, which were even lower than seen for ethambutol (MIC, $15.3 \mu \mathrm{M}$ ). More importantly, these compounds showed MICs of $10 \mu \mathrm{M}$ against extensively drug resistant tuberculosis strains. Due to the problematic cardiotoxicity reported previously for inhibitors with an aminopiperidine linker, hERG K-channel inhibition by these compounds was investigated with the zebra fish model; this showed that they had a promising safety profile in comparison with the control.

Based on previous structure-based in-silico pharmacophore modelling studies that identified the $N$-(3-carbamoyl-5-phenylthiophen-2-yl)thiophene-2-carboxamide $\mathbf{3 4}$ as the most promising lead [76], novel phenylthiophen-based inhibitors of $M$. tuberculosis GyrB were obtained through structure-based drug design [77]. A library of 28 analogues was synthesized to obtain tractable SARs and to improve the activities. Five of these analogues indeed showed activities in the GyrB ATPase assay, with $\mathrm{IC}_{50}<5$ $\mu \mathrm{M}$.

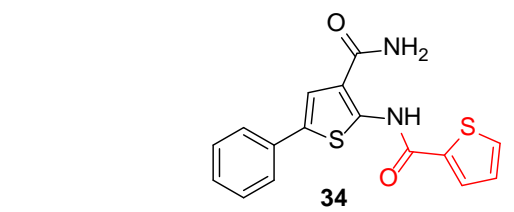

M.tuberculosis DNA gyrase supercoiling $\mathrm{IC}_{50}=1.16 \pm 0.25 \mu \mathrm{M}$ M.smegmatis GyrB IC50



$35 \mathrm{X}=\mathrm{O} ; \mathrm{R}=4$-chlorophenyl; $\mathrm{IC}=3.14 \pm 0.76 \mu \mathrm{M}$ $36 \mathrm{X}=\mathrm{O} ; \mathrm{R}=$ phenyl; $37 \mathrm{X}=\mathrm{S} ; \mathrm{R}=4$-toluyl;
$\mathrm{IC}_{50}=2.46 \pm 0.56 \mu \mathrm{M}$



$38 \mathrm{R}=$ phenyl; $\quad \mathrm{IC}_{50}=0.86 \pm 0.81 \mu \mathrm{M}$ $39 \mathrm{R}=\mathrm{n}$-butyryl; $\mathrm{IC}_{50}$

Figure 13. Structures and activities of the phenylthiophen-based mycobacterial inhibitors [76, 77]. 
Urea, thiourea and amide derivatives were then prepared, and phenyl substitution was shown to provide the most potent compound 38, with an $\mathrm{IC}_{50}$ of $0.86 \mu \mathrm{M}$ in the GyrB ATPase assay, and an $\mathrm{IC}_{50}$ of $0.76 \mu \mathrm{M}$ in the supercoiling assay on M. tuberculosis DNA gyrase. All of the compounds prepared were also tested for in-vitro antitubercular activity, and compound $\mathbf{3 8}$ again showed the best activity (MIC, $4.8 \mu \mathrm{M}$ ). Differential scanning fluorimetry also showed better thermal stabilization of the protein-ligand complex, with a $T_{m}$ shift of $2.5^{\circ} \mathrm{C}$.

\section{Preclinical Optimization strategies}

As several compounds entered into the more mature stages of preclinical development, both their activities and their other properties were optimised. Perola et al. from Vertex [78] studied the role of plasma and tissue protein binding on the activities of GyrB inhibitors. Studies of the benzimidazole ureas showed $95 \%$ protein binding and 16-fold to 128-fold serum shifts (i.e., ratio between MIC in the presence and absence of 50\% human serum). Non-specific hydrophobic interactions and specific binding with plasma proteins are both important for serum protein binding. Acidic compounds show greater levels of protein binding than neutral compounds, which in turn show more protein binding than basic structures or zwitterions. Basic groups were thus introduced into the lead compounds, with reductions in the serum shifts observed in the majority of cases. However, this was accompanied by increased clearance and overall worse intravenous pharmacokinetics in rats, and thus the basic groups incorporated were changed to neutral analogues. Also, the commonly used protein binding prediction models were not adequate here, and therefore a novel model was designed using a reduced library of 202 compounds. The correlations between serum shift, molecular weight, $\operatorname{cog} P$ and polar surface area were determined. Then further analysis of two subclasses, A and B, established the criteria for the optimal properties necessary to reduce the serum shift (Fig. 14).

This new strategy prompted the synthesis of 61 additional compounds of both of these subclasses. The majority of these had low nanomolar activities against GyrB and topoisomerase IV, with MICs against $S$. aureus in the range of $0.016 \mu \mathrm{g} / \mathrm{mL}$ to $0.25 \mu \mathrm{g} / \mathrm{mL}$. The serum shifts correlated with the predicted values in $75 \%$ of cases, with low-shift compounds predicted with $62 \%$ accuracy, and highshift compounds with 92\% accuracy. Compound 40 (Fig. 14) showed an eight-fold reduced serum shift, a MIC (S. aureus) of $0.031 \mu \mathrm{g} / \mathrm{mL}$, and improved in-vivo efficacy. This study showed that the promising prediction model was useful for the design of compounds with low protein binding and with increased in-vivo properties. 



Figure 14. The two subclasses of benzimidazole ureas, with their optimal properties for reduced serum shift [78-80].

Previously reported aminobenzimidazole analogues were also optimized using structure-guided strategies for the promotion of their antimicrobial activities [30]. However, some of the compounds had labile safety profiles due to interactions with liver proteins, and thus a second generation of inhibitors was obtained using a metabolic shift strategy [79]. A new preclinical candidate 41 (Fig. 14; known as VXc-486) from Vertex Pharmaceuticals was reported, and then further optimized by the phosphate prodrug approach. The efficacies of $\mathbf{4 1}$ and its phosphate prodrug against $M$. tuberculosis and nontuberculosis mycobacteria were explored both in-vitro and in-vivo by Locher et al. [80]. Here, $\mathbf{4 1}$ showed antimycobacterial potency against drug-sensitive and drug-resistant strains of $M$. tuberculosis, with MICs from $0.05 \mu \mathrm{g} / \mathrm{mL}$ to $0.5 \mu \mathrm{g} / \mathrm{mL}$ under the different culture conditions (e.g., broth, macrophages). Moreover, the activities of $\mathbf{4 1}$ against multiple drug resistant, extensive drug resistant, and genetically diverse M. tuberculosis and non-tuberculosis strains supported its potential for treatment of drug resistant M. tuberculosis. Exploration of the resistance profile of $\mathbf{4 1}$ included the A92S (introduction of hydroxyl into the ATP binding site) and S208A (switch of serine to alanine, with altered H-bond network) mutations reported previously. In-vivo studies also showed the potency of $\mathbf{4 1}$ in the form of its phosphate prodrug. Furthermore, promising results were obtained for $\mathbf{4 1}$ in combination with rifapentine, pyrazinamide, linezolid and bedaquiline, and in in-vivo models of drug-sensitive and drugresistant tuberculosis. The use of $\mathbf{4 1}$ instead of moxifloxacin might thus be considered following some further studies. Spero Therapeutics acquired right over compound VXc-486 (41) and it is continuing its clinical development under name SPR720.

\section{Novel structural insights}

Although ATP binding to GyrB has been thoroughly studied and described, when the structure of the 43-kDa domain of GyrB with the ATP analogue adenosine-5' - ( $\beta$, $\gamma$-imido) triphosphate (AMPPNP) was solved [20], Stanger et al. defined other conformational states during the hydrolysis reaction route [81]. 
Structures were solved for the 43-kDa domain of GyrB in complex with the pre-hydrolysis analogue $\mathrm{ADP} \cdot \mathrm{BeF}_{3}$, the post-hydrolysis analogue $\mathrm{ADP}-\mathrm{P}_{\mathrm{i}}$, and the product ADP (PDB: 4PRV, 4PRX, 4PU9, 4R1F), to better understand the ATP hydrolysis coordination and the passage of the DNA strand. In comparison to AMPPNP, the post-hydrolysis state showed extensive domain reorientation. Structures were obtained using the molecular replacement method with the complex with AMPPNP as the search model. Structural comparisons confirmed the virtual identity of both the transducer and the ATPase domain, with root mean square deviations from $0.32 \AA$ to $0.57 \AA$, although the transducer domain was reoriented by $12^{\circ}$. It has been shown that the compulsory post-hydrolysis state characterized by increased distance between the $\gamma$-phosphate and the $\beta$-phosphate was achieved prior to phosphate release, which induces the enzymatic cycle.

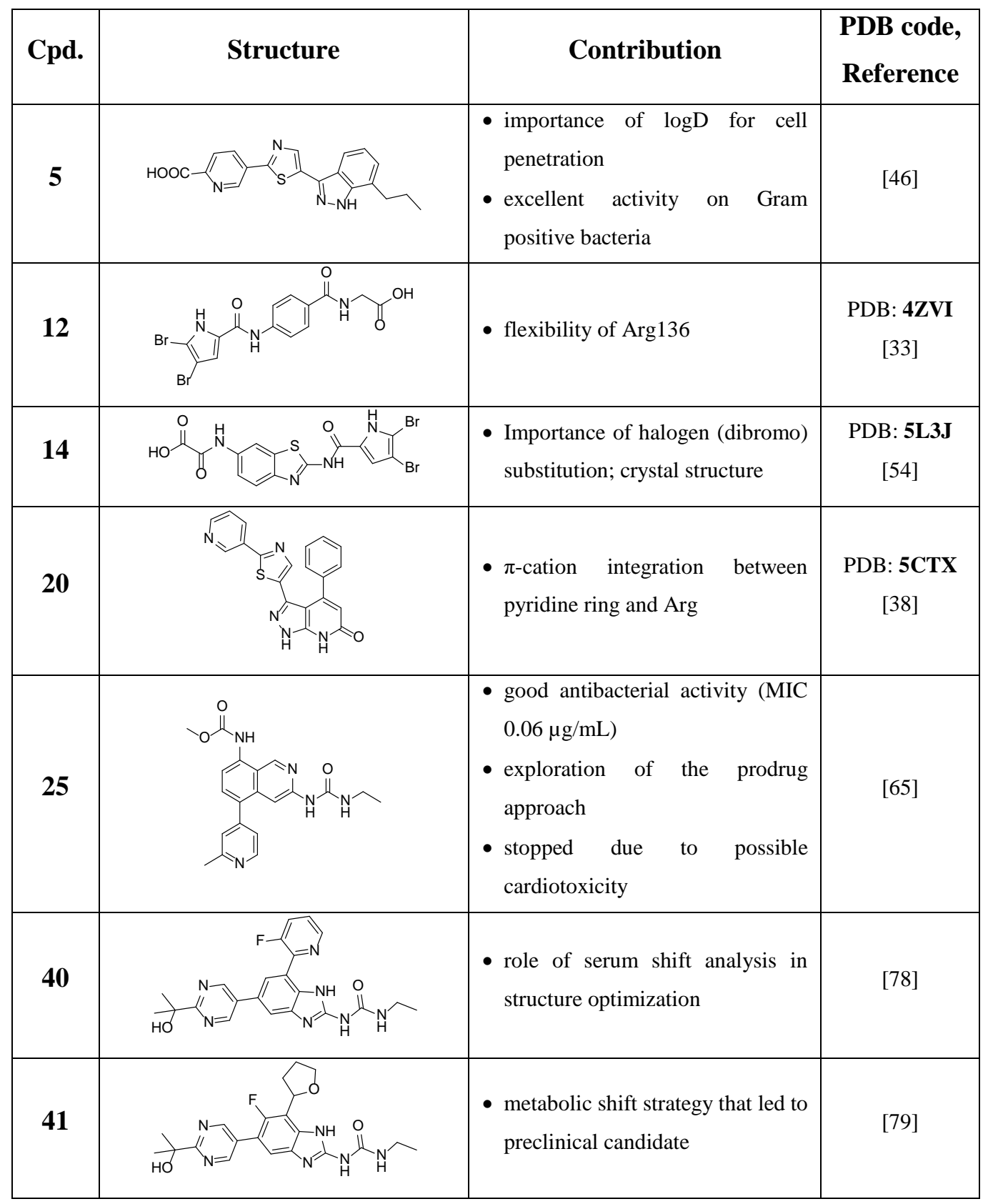


Figure 15. Seven compounds that have contributed to the development of GyrB inhibitors in recent years.

\section{Conclusions}

The successful discovery of novel antibacterial classes is urgently required, as bacterial resistance will continue as an increasing threat for the forthcoming years. Topoisomerases are attractive targets for drug discovery, as they are well-established enzymes that are conserved across the broad spectrum of bacterial species. The structural and functional similarities between DNA gyrase and topoisomerase IV indicate the possibility of dual targeting. Here, we have presented an overview of the recent developments in the discovery of GyrB/ParE inhibitors.

The main focus of recent research in this field has been on improvement of enzymatic affinities via optimized binding, broadening of the bacterial profile towards Gram-negative bacteria, and improvement of the physico-chemical properties and pharmacokinetic profiles of potential drug candidates. Some of the GyrB inhibitors presented here are also substrates for efflux pumps, and as a consequence, their optimal antibacterial activities have not been achieved. If this issue can be overcome then this would increase the chances of the development of potent inhibitors. The field of discovery of novel GyrB/ParE inhibitors is viable and remains very challenging and difficult. Indeed, in 2015, Bisacchi and Manchester [16] used word "fragile" to describing the state of antibacterial research and development within the pharmaceutical industry; we believe that the same word still best describes this today.

\section{Future perspectives}

Extensive research on gyrase and bacterial topoisomerase IV inhibitors is likely to result in novel drug candidates for patients. The field of novel GyrB/ParE ATP-competitive inhibitors is a particularly promising one, as bacterial resistance is less likely to develop. From the medicinal point of view, GyrB and ParE are validated and well-explored targets, and on-going activities are focused on the late stages of preclinical activity, with excellent clinical candidates more recently acquired. As there are several initiatives that connect industrial and governmental/ academic efforts (e.g., IMI), these also represent potential pathways for new drugs to reach the market.

\section{Executive summary}

- Topoisomerases II are essential enzymes that alter DNA topology through cleaving of the two DNA strands. 
- DNA gyrase and topoisomerase IV are heterotetramers that are structurally and functionally similar, to potentially allow dual targeting. DNA gyrase introduces negative supercoils, while topoisomerase IV is involved in chromosome decatenation during replication.

- The GyrA/ParC subunits are targeted by fluoroquinolones, as well established inhibitors that have increasing bacterial resistance issues.

- After the withdrawal of the only marketed GyrB inhibitor, novobiocin, from the market, no other inhibitors have passed phase I clinical trials, and currently there are no GyrB/ParE inhibitors on the market.

- Recently reported structural classes (e.g., benzimidazoles, pyrrolamides, pyrrolopyrimidines, pyridylureas, pyrazolopyridones) share the motif of the H-bond donor-acceptor network with Asp73, with the aromatic moiety that forms $\pi$-cation interactions with arginine residues.

- Several of the approaches to GyrB inhibitor discovery are described here, with the search for novel structural classes and the improvement of those already known, regarding enzyme affinity, antibacterial activity, and pharmokinetic properties.

- This review includes 18 new co-crystal structures of various GyrB domains and ligands.

- As well as the preferential targeting of inhibitors to Gram-negative pathogens, mycobacterial inhibitors are also being examined.

- A relatively frequent problem of current research includes efflux pumps and their actions on potential GyrB inhibitors as substrates. Solving this problem might move the field forward.

- Future efforts in the discovery of GyrB inhibitors need to define potent candidates with balanced invitro and in-vivo properties, together with a suitable pharmacokinetic profile. These then need to progress to clinical trials. 


\section{References}

1. Brown ED, Wright GD. Antibacterial drug discovery in the resistance era. Nature 529(7586), 336-343 (2016).

2. Who. Antimicrobial resistance. http://www.who.int/topics/antimicrobial_resistance/en/

3. Silver LL. Challenges of antibacterial discovery. Clin. Microbiol. Rev. 24(1), 71-109 (2011).

4. Walsh CT, Wencewicz TA. Prospects for new antibiotics: a molecule-centered perspective. J. Antibiot. 67(1), 7-22 (2014).

5. East SP, Silver LL. Multitarget ligands in antibacterial research: progress and opportunities. Expert Opin. Drug. Discov. 8(2), 143-156 (2013).

6. Forterre P, Gribaldo S, Gadelle D, Serre MC. Origin and evolution of DNA topoisomerases. Biochimie 89(4), 427-446 (2007).

7. Gellert M, O'dea MH, Itoh T, Tomizawa J. Novobiocin and coumermycin inhibit DNA supercoiling catalyzed by DNA gyrase. Proc. Natl. Acad. Sci. USA 73(12), 4474-4478 (1976).

8. Champoux JJ. DNA topoisomerases: Structure, function, and mechanism. Annu Rev Biochem. 70 369-413 (2001).

-Comprehensive overview of topoisomerases (type I and II) and their characteristics.

9. Dutta R, Inouye M. GHKL, an emergent ATPase/kinase superfamily. Trends Biochem. Sci. 25(1), 24-28 (2000).

10. Burlison JA, Neckers L, Smith AB, Maxwell A, Blagg BS. Novobiocin: redesigning a DNA gyrase inhibitor for selective inhibition of hsp90. J. Am. Chem. Soc. 128(48), 15529-15536 (2006).

11. Donnelly AC, Zhao H, Reddy Kusuma B, Blagg BSJ. Cytotoxic sugar analogues of an optimized novobiocin scaffold. MedChemComm 1(2), 165-170 (2010).

12. Byrd KM, Subramanian C, Sanchez J et al. Synthesis and biological evaluation of novobiocin core analogues as Hsp90 inhibitors. Chem. Eur. J. 22(20), 6921-6931 (2016).

13. Lillsunde KE, Tomasic T, Kikelj D, Tammela P. Marine alkaloid oroidin analogues with antiviral potential: A novel class of synthetic compounds targeting the cellular chaperone Hsp90. Chem. Biol. Drug Des. 90(6), 1147-1154 (2017). 
14. Emmerson AM, Jones AM. The quinolones: decades of development and use. $J$. Antimicrob. Chemother. 51 Suppl 1 13-20 (2003).

15. Federal Register US. https://www.federalregister.gov/documents/2011/01/19/20111000/determination-that-albamycin-novobiocin-sodium-capsule-250-milligrams-waswithdrawn-from-sale-for

16. Bisacchi GS, Manchester JI. A new-class antibacterial - almost. Lessons in drug discovery and development: a critical analysis of more than 50 years of effort toward ATPase inhibitors of DNA gyrase and topoisomerase IV. ACS Infect. Dis. 1(1), 4-41 (2015).

••Excellent comprehensive review that describes the history of ATP-competitive DNA gyrase and topoisomerase IV inhibitors

17. Holdgate GA, Tunnicliffe A, Ward WHJ et al. The entropic penalty of ordered water accounts for weaker binding of the antibiotic novobiocin to a resistant mutant of DNA gyrase: a thermodynamic and crystallographic study. Biochem. 36(32), 9663-9673 (1997).

18. Angehrn P, Buchmann S, Funk C et al. New antibacterial agents derived from the DNA gyrase inhibitor cyclothialidine. J. Med. Chem. 47(6), 1487-1513 (2004).

19. Heide L. New aminocoumarin antibiotics as gyrase inhibitors. Int. J. Med. Microbiol. 304(1), 31-36 (2014).

20. Wigley DB, Davies GJ, Dodson EJ, Maxwell A, Dodson G. Crystal structure of an Nterminal fragment of the DNA gyrase B protein. Nature 351(6328), 624-629 (1991).

-First exploration of the GyrB crystal structure.

21. Lewis RJ, Singh OM, Smith CV et al. The nature of inhibition of DNA gyrase by the coumarins and the cyclothialidines revealed by X-ray crystallography. EMBO J 15(6), 1412-1420 (1996).

22. Skedelj V, Tomasic T, Masic LP, Zega A. ATP-binding site of bacterial enzymes as a target for antibacterial drug design. J. Med. Chem. 54(4), 915-929 (2011).

23. Tohyama S, Takahashi Y, Akamatsu Y. Biosynthesis of amycolamicin: the biosynthetic origin of a branched alpha-aminoethyl moiety in the unusual sugar amycolose. $J$. Antibiot. (Tokyo) 63(3), 147-149 (2010).

24. Sawa R, Takahashi Y, Hashizume H et al. Amycolamicin: a novel broad-spectrum antibiotic inhibiting bacterial topoisomerase. Chemistry 18(49), 15772-15781 (2012). 
25. Phillips JW, Goetz MA, Smith SK et al. Discovery of kibdelomycin, a potent new class of bacterial type II topoisomerase inhibitor by chemical-genetic profiling in Staphylococcus aureus. Chem. Biol. 18(8), 955-965 (2011).

26. Lu J, Patel S, Sharma N et al. Structures of kibdelomycin bound to Staphylococcus aureus GyrB and ParE showed a novel U-shaped binding mode. ACS Chem. Biol. 9(9), 2023-2031 (2014).

27. Singh SB, Goetz MA, Smith SK et al. Kibdelomycin A, a congener of kibdelomycin, derivatives and their antibacterial activities. Bioorg. Med. Chem. Lett. 22(23), 71277130 (2012).

28. Edwards MJ, Flatman RH, Mitchenall LA et al. A crystal structure of the bifunctional antibiotic simocyclinone D8, bound to DNA gyrase. Science 326(5958), 1415-1418 (2009).

29. Silver LL. Are natural products still the best source for antibacterial discovery? The bacterial entry factor. Expert Opin. Drug Discov. 3(5), 487-500 (2008).

30. Charifson PS, Grillot A-L, Grossman TH et al. Novel dual-targeting benzimidazole urea inhibitors of DNA gyrase and topoisomerase IV possessing potent antibacterial activity: intelligent design and evolution through the judicious use of structure-guided design and stucture-activity relationships. J. Med. Chem. 51(17), 5243-5263 (2008).

31. Sherer BA, Hull K, Green O et al. Pyrrolamide DNA gyrase inhibitors: Optimization of antibacterial activity and efficacy. Bioorg. Med. Chem. Lett. 21(24), 7416-7420 (2011).

32. Zidar N, Tomašic T, Macut H et al. New N-phenyl-4,5-dibromopyrrolamides and NPhenylindolamides as ATPase inhibitors of DNA gyrase. Eur. J. Med. Chem. 117 197211 (2016).

33. Zidar N, Macut $\mathrm{H}$, Tomasic $\mathrm{T}$ et al. N-Phenyl-4,5-dibromopyrrolamides and Nphenylindolamides as ATP-competitive DNA gyrase B inhibitors: design, synthesis, and evaluation. J. Med. Chem. 58(15), 6179-6194 (2015).

- Comprehensive study that describes the discovery of a novel scaffold, including the cocrystal structure of the ligand with GyrB

34. Tomašič T, Mirt M, Barančoková M et al. Design, synthesis and biological evaluation of 4,5-dibromo-N-(thiazol-2-yl)-1H-pyrrole-2-carboxamide derivatives as novel DNA gyrase inhibitors. Bioorg. Med. Chem. 25(1), 338-349 (2017).

35. Tari LW, Trzoss M, Bensen DC et al. Pyrrolopyrimidine inhibitors of DNA gyrase B (GyrB) and topoisomerase IV (ParE). Part I: Structure guided discovery and 
optimization of dual targeting agents with potent, broad-spectrum enzymatic activity. Bioorg. Med. Chem. Lett. 23(5), 1529-1536 (2013).

36. Bensen D, Chen Z, Creighton C et al: WO032050 A3 (2012).

37. Basarab GS, Manchester JI, Bist S et al. Fragment-to-hit-to-lead discovery of a novel pyridylurea scaffold of ATP-competitive dual targeting type II topoisomerase inhibiting antibacterial agents. J. Med. Chem. 56(21), 8712-8735 (2013).

38. Mesleh MF, Cross JB, Zhang $\mathrm{J}$ et al. Fragment-based discovery of DNA gyrase inhibitors targeting the ATPase subunit of GyrB. Bioorg. Med. Chem. Lett. 26(4), 13141318 (2016).

39. Cross JB, Zhang J, Yang Q et al. Discovery of pyrazolopyridones as a novel class of gyrase B inhibitors using structure-guided design. ACS Med. Chem. Lett. 7(4), 374-378 (2016).

40. Boehm HJ, Boehringer M, Bur D et al. Novel inhibitors of DNA gyrase: 3D structure based biased needle screening, hit validation by biophysical methods, and 3D guided optimization. A promising alternative to random screening. J. Med. Chem. 43(14), 2664-2674 (2000).

41. Mayer C, Janin YL. Non-quinolone inhibitors of bacterial type IIA topoisomerases: a feat of bioisosterism. Chem. Rev. 114(4), 2313-2342 (2014).

42. Tse-Dinh YC. Targeting bacterial topoisomerases: how to counter mechanisms of resistance. Future Med. Chem. 8(10), 1085-1100 (2016).

- Overview of topoisomerases as targets of drug design and the associated resistance issues and their mechanisms.

43. Nagaraja V, Godbole AA, Henderson SR, Maxwell A. DNA topoisomerase I and DNA gyrase as targets for TB therapy. Drug Discov. Today 22(3), 510-518 (2017).

44. Li X, Tari L, Bensen DC, Trzoss M, Lam T, Zhang J et al. Poster F-2017. Presented at: 52nd Interscience Conference on Antimicrobial Agents and Chemotherapy. San Francisco, CA, USA, 7-11 December 2012.

45. Bensen D, Borchardt A, Chen Z et al: WO043272 A1 (2014).

46. Zhang J, Yang Q, Romero JaC et al. Discovery of indazole derivatives as a novel class of bacterial gyrase B inhibitors. ACS Med. Chem. Lett. 6(10), 1080-1085 (2015).

47. Zhang J, Yang Q, Cross JB et al. Discovery of azaindole ureas as a novel class of bacterial gyrase B inhibitors. J. Med. Chem. 58(21), 8503-8512 (2015).

• Comprehensive study describing the discovery of a novel scaffold, including co-crystal structure of the ligand with GyrB. 
48. Tomašič T, Mašič LP. Prospects for developing new antibacterials targeting bacterial type IIA topoisomerases. Curr. Top. Med. Chem. 14(1), 130-151 (2014).

49. Schrodinger, Llc. The PyMOL Molecular Graphics System, Version 1.8. (2015).

50. Zidar N, Montalvão S, Hodnik Ž et al. Antimicrobial activity of the marine alkaloids, clathrodin and oroidin, and their synthetic analogues. Mar. Drugs 12(2), 940-963 (2014).

51. Tomašič T, Katsamakas S, Hodnik Ž et al. Discovery of 4,5,6,7-tetrahydrobenzo[1,2d]thiazoles as novel DNA gyrase inhibitors targeting the ATP-binding site. J. Med. Chem. 58(14), 5501-5521 (2015).

52. Eakin AE, Green O, Hales N et al. Pyrrolamide DNA gyrase inhibitors: fragment-based nuclear magnetic resonance screening to identify antibacterial agents. Antimicrob. Agents Chemother. 56(3), 1240-1246 (2012).

53. Uria-Nickelsen M, Blodgett A, Kamp H, Eakin A, Sherer B, Green O. Novel DNA gyrase inhibitors: microbiological characterisation of pyrrolamides. Int. J. Antimicrob. Agents 41(1), 28-35 (2013).

54. Gjorgjieva M, Tomašič T, Barančokova $\mathrm{M}$ et al. Discovery of benzothiazole scaffoldbased DNA gyrase B inhibitors. J. Med. Chem. 59(19), 8941-8954 (2016).

• Comprehensive study that describes the discovery of a novel scaffold, including the cocrystal structure of the ligand with GyrB

55. Bellon S, Parsons JD, Wei Y et al. Crystal structures of Escherichia coli topoisomerase IV ParE subunit (24 and 43 kilodaltons): a single residue dictates differences in novobiocin potency against topoisomerase IV and DNA gyrase. Antimicrob. Agents Chemother. 48(5), 1856-1864 (2004).

56. Durcik M, Tammela P, Barancokova $\mathrm{M}$ et al. Synthesis and evaluation of $\mathrm{N}$ phenylpyrrolamides as DNA gyrase $\mathrm{B}$ inhibitors. ChemMedChem doi:10.1002/cmdc.201700549 (2017).

57. Jukić M, Ilaš J, Brvar M, Kikelj D, Cesar J, Anderluh M. Linker-switch approach towards new ATP binding site inhibitors of DNA gyrase B. Eur. J. Med. Chem. 125 500-514 (2017).

58. Cotman AE, Trampuž M, Brvar M et al. Design, synthesis, and evaluation of novel tyrosine-based DNA gyrase B inhibitors. Arch. Pharm. 350(8), 1700087-n/a (2017).

59. Tomašič T, Barancokova M, Zidar N, Ilas J, Tammela P, Kikelj D. Design, synthesis, and biological evaluation of 1-ethyl-3-(thiazol-2-yl)urea derivatives as Escherichia coli DNA gyrase inhibitors. Arch. Pharm. 351(1), (2018). 
60. Jakopin Ž, Ilaš J, Barančoková M et al. Discovery of substituted oxadiazoles as a novel scaffold for DNA gyrase inhibitors. Eur. J. Med. Chem. 130 171-184 (2017).

61. Soneda T, Takeshita H, Kagoshima Y et al: US8927588 B2 (2015).

62. Khera MK, Mathur T, Sattigeri JA et al: WO056012 A1 (2017).

63. Keserü GM, Erlanson DA, Ferenczy GG, Hann MM, Murray CW, Pickett SD. Design principles for fragment libraries: maximizing the value of learnings from pharma fragment-based drug discovery (FBDD) programs for use in academia. J. Med. Chem. 59(18), 8189-8206 (2016).

64. Rees DC, Congreve M, Murray CW, Carr R. Fragment-based lead discovery. Nat. Rev. Drug Discov. 3(8), 660-672 (2004).

65. Panchaud $\mathrm{P}$, Bruyère $\mathrm{T}$, Blumstein A-C et al. Discovery and optimization of isoquinoline ethyl ureas as antibacterial agents. J. Med. Chem. 60(9), 3755-3775 (2017).

• Comprehensive study that describes the discovery of lead optimisation, including the cocrystal structure of the ligand with GyrB.

66. East SP, Czaplewski LG, Haydon DJ. Ethyl urea inhibitors of the bacterial type II topoisomerases DNA gyrase (GyrB) and topoisomerase IV (ParE). In: Designing MultiTarget Drugs, The Royal Society of Chemistry Cambridge, UK 335-352 (2012).

67. Cole ST, Brosch R, Parkhill J et al. Deciphering the biology of Mycobacterium tuberculosis from the complete genome sequence. Nature 393(6685), 537-544 (1998).

68. Manjunatha UH, Madhusudan K, Visweswariah SS, Nagaraja V. Structural heterogeneity in DNA gyrases from Gram-positive and Gram-negative bacteria. Curr. Sci. 79(7), 968-974 (2000).

69. T Takiff HE, Salazar L, Guerrero $\mathrm{C}$ et al. Cloning and nucleotide sequence of Mycobacterium tuberculosis gyrA and gyrB genes and detection of quinolone resistance mutations. Antimicrob. Agents Chemother. 38(4), 773-780 (1994).

70. Manjunatha UH, Dalal M, Chatterji M, Radha DR, Visweswariah SS, Nagaraja V. Functional characterisation of mycobacterial DNA gyrase: an efficient decatenase. Nucleic Acids Res. 30(10), 2144-2153 (2002).

71. Reddy KI, Srihari K, Renuka J et al. An efficient synthesis and biological screening of benzofuran and benzo[d]isothiazole derivatives for Mycobacterium tuberculosis DNA GyrB inhibition. Bioorg. Med. Chem. 22(23), 6552-6563 (2014).

72. Jeankumar VU, Renuka J, Pulla VK et al. Development of novel N-linked aminopiperidine-based mycobacterial DNA gyrase B inhibitors: Scaffold hopping from known antibacterial leads. Int. J. Antimicrob. Agents 43(3), 269-278 (2014). 
73. Bax BD, Chan PF, Eggleston DS et al. Type IIA topoisomerase inhibition by a new class of antibacterial agents. Nature 466(7309), 935-940 (2010).

74. Reck F, Alm R, Brassil P et al. Novel N-linked aminopiperidine inhibitors of bacterial topoisomerase type II: broad-spectrum antibacterial agents with reduced hERG activity. Journal of Medicinal Chemistry 54(22), $7834-7847$ (2011).

75. Reck F, Alm RA, Brassil P et al. Novel N-linked aminopiperidine inhibitors of bacterial topoisomerase type II with reduced $\mathrm{pK}(\mathrm{a})$ : antibacterial agents with an improved safety profile. J. Med. Chem. 55(15), 6916-6933 (2012).

76. Saxena S, Renuka J, Yogeeswari P, Sriram D. Discovery of novel mycobacterial DNA gyrase B inhibitors: in-silico and in-vitro biological evaluation. Mol. Inform. 33(9), 597609 (2014).

77. Saxena S, Samala G, Renuka J, Sridevi JP, Yogeeswari P, Sriram D. Development of 2-amino-5-phenylthiophene-3-carboxamide derivatives as novel inhibitors of Mycobacterium tuberculosis DNA GyrB domain. Bioorg. Med. Chem. 23(7), 14021412 (2015).

78. Perola E, Stamos D, Grillot A-L et al. Successful application of serum shift prediction models to the design of dual targeting inhibitors of bacterial gyrase $\mathrm{B}$ and topoisomerase IV with improved in-vivo efficacy. Bioorg. Med. Chem. Lett. 24(9), 2177-2181 (2014).

••Design of serum shift prediction models for evaluation of in-vivo efficacy of gyrase inhibitors.

79. Grillot A-L, Tiran AL, Shannon D et al. Second-generation antibacterial benzimidazole ureas: discovery of a preclinical candidate with reduced metabolic liability. J. Med. Chem. 57(21), 8792-8816 (2014).

80. Locher CP, Jones SM, Hanzelka BL et al. A novel inhibitor of gyrase B is a potent drug candidate for treatment of tuberculosis and nontuberculosis mycobacterial infections. Antimicrob. Agents Chemother. 59(3), 1455-1465 (2015).

••Evaluation of antimycobacterial inhibitor with improved safety profile, as a preclinical candidate.

81. Stanger FV, Dehio C, Schirmer T. Structure of the N-terminal gyrase B fragment in complex with ADP.Pi reveals rigid-body motion induced by ATP hydrolysis. PLoS ONE 9(9), e107289 (2014).

-Definition of the mechanism and enzyme conformation during ATP hydrolysis. 